\title{
TRPC3-Nox2 complex mediates doxorubicin-induced myocardial atrophy
}

\author{
Tsukasa Shimauchi, ${ }^{1,2,3}$ Takuro Numaga-Tomita, ${ }^{1,4}$ Tomoya Ito, ${ }^{1}$ Akiyuki Nishimura, ${ }^{1,4}$ \\ Ryosuke Matsukane, ${ }^{1,2}$ Sayaka Oda, ${ }^{1,4}$ Sumio Hoka, ${ }^{3}$ Tomomi Ide, ${ }^{5}$ Norimichi Koitabashi, ${ }^{6}$ \\ Koji Uchida, ${ }^{7}$ Hideki Sumimoto, ${ }^{8}$ Yasuo Mori, ${ }^{9}$ and Motohiro Nishida ${ }^{1,2,4,10}$ \\ 'Division of Cardiocirculatory Signaling, Okazaki Institute for Integrative Bioscience (National Institute for Physiological \\ Sciences), National Institutes of Natural Sciences, Aichi, Japan. 'Department of Translational Pharmaceutical Sciences, \\ Graduate School of Pharmaceutical Sciences, and ${ }^{3}$ Department of Anesthesiology and Critical Care Medicine, Graduate \\ School of Medical Sciences, Kyushu University, Fukuoka, Japan. ${ }^{4}$ School of Life Science, The Graduate University for \\ Advanced Studies (SOKENDAI), Department of Physiological Sciences, Aichi, Japan. ${ }^{5}$ Department of Cardiovascular \\ Medicine, Graduate School of Medical Sciences, Kyushu University, Fukuoka, Japan. ${ }^{6}$ Department of Medicine and \\ Biological Sciences, Graduate School of Medicine, Gunma University, Gunma, Japan. ${ }^{7}$ Department of Applied Biological \\ Chemistry, Graduate School of Agricultural and Life Sciences, The University of Tokyo, Tokyo, Japan. ${ }^{8}$ Department of \\ Biochemistry, Graduate School of Medical Sciences, Kyushu University, Fukuoka, Japan. ${ }^{9}$ Department of Synthetic \\ Chemistry and Biological Chemistry, Graduate School of Engineering, Kyoto University, Kyoto, Japan. \\ ${ }^{10}$ PRESTO, JST, 4-1-8 Honcho, Kawaguchi, Saitama, Japan.
}

Myocardial atrophy is a wasting of cardiac muscle due to hemodynamic unloading. Doxorubicin is a highly effective anticancer agent but also induces myocardial atrophy through a largely unknown mechanism. Here, we demonstrate that inhibiting transient receptor potential canonical 3 (TRPC3) channels abolishes doxorubicin-induced myocardial atrophy in mice. Doxorubicin increased production of ROS in rodent cardiomyocytes through hypoxic stress-mediated upregulation of NADPH oxidase 2 (Nox2), which formed a stable complex with TRPC3. Cardiomyocyte-specific expression of TRPC3 C-terminal minipeptide inhibited TRPC3-Nox2 coupling and suppressed doxorubicin-induced reduction of myocardial cell size and left ventricular (LV) dysfunction, along with its upregulation of Nox2 and oxidative stress, without reducing hypoxic stress. Voluntary exercise, an effective treatment to prevent doxorubicin-induced cardiotoxicity, also downregulated the TRPC3-Nox2 complex and promoted volume load-induced LV compliance, as demonstrated in TRPC3-deficient hearts. These results illustrate the impact of TRPC3 on LV compliance and flexibility and, focusing on the TRPC3-Nox2 complex, provide a strategy for prevention of doxorubicin-induced cardiomyopathy.

Conflict of interest: The authors have declared that no conflict of interest exists.

Submitted: February 10, 2017

Accepted: June 27, 2017

Published: August 3, 2017

\section{Reference information:}

JCI Insight. 2017;2(15):e93358.

https://doi.org/10.1172/jci.

insight.93358.

\section{Introduction}

Cardiac plasticity is defined as structural remodeling of the heart in response to environmental demands and is an intrinsic compensatory mechanism to reduce hemodynamic workload $(1,2)$. Exercise, pregnancy, and postnatal growth all promote physiologic myocardial growth, whereas neurohumoral activation, hypertension, and myocardial injury can all cause pathological hypertrophy, which increases the risk of heart failure by reducing myocardial flexibility through myocardial stiffening. Cardiac atrophy, defined as an acquired reduction in the size and mass of the heart, is a complication of protracted bed rest, prolonged weightlessness during space travel, and mechanical unloading through the use of a ventricular assist device. The atrophied myocardium also exhibits reduced contractility due to decreased myocardial flexibility. Why myocardial flexibility is reduced during the processes of both cardiac atrophy and pathological hypertrophy is largely unknown.

Doxorubicin (DOX) is a highly effective anticancer agent used to treat a variety of hematologic and solid malignancies (3-6). However, its dose-dependent cardiotoxicity limits its clinical use (3-6). The cardiotoxic effects of DOX range from asymptomatic increases in left ventricular (LV) wall stress to reductions in ejection fraction, arrhythmias, and highly symptomatic congestive heart failure, which are all associated with high mortality $(6,7)$. DOX initially forces the heart to shrink, which leads to induction of myocardial 
apoptosis and interstitial fibrosis at later stages of LV dilated cardiomyopathy $(8,9)$. There is growing evidence that the DOX-induced cardiac apoptosis and fibrosis are mainly caused by excess production of ROS (10-13), which is initiated by NADPH oxidase (Nox) activation $(13,14)$.

Two Nox enzymes, Nox2 and Nox4, are predominantly expressed in mammalian hearts (15-17). Nox 2 is activated by angiotensin II, endothelin- 1 , tumor necrosis factor- $\beta$, growth factors, and mechanical force, and Nox2-dependent ROS production reportedly mediates congestive heart failure in humans (18). Nox2 also participates in mechanochemical transduction in hearts through ROS production during LV diastolic filling (i.e., mechanical stretch of cardiomyocytes) $(19,20)$. Notably, Nox2-deficient mice reportedly exhibit resistance to DOX-induced cardiac atrophy (13), but how Nox2 contributes to DOX-induced myocardial atrophy remains obscure.

Transient receptor potential (TRP) channels, originally identified as a visual phototransduction mutant, have been attracting attention as multimodal cation channels regulated by several environmental factors, including mechanical stress, in vertebrates (21). In particular, two TRP canonical (TRPC) subfamily members, TRPC3 and TRPC6, reportedly participate in the development of pathological hypertrophy caused by neurohumoral factors $(22,23)$ and mechanical stress $(24-26)$. Our recent studies using TRPC-KO mice revealed that TRPC3, but not TRPC6, functions as a positive regulator of ROS, leading to induction of mechanical stress-induced maladaptive fibrosis $(15,20,27)$. TRPC3 interacts with Nox2 via TRPC3-specific C-terminal sites, thereby protecting Nox2 from proteasome-dependent degradation and amplifying $\mathrm{Ca}^{2+}$-dependent Nox2 activation through TRPC3-mediated background $\mathrm{Ca}^{2+}$ entry (20). Nox2 also stabilizes TRPC3 proteins to enhance mechanical stress-induced background $\mathrm{Ca}^{2+}$ entry into cardiomyocytes. Whether the TRPC3-Nox2 axis participates in DOX-induced myocardial atrophy is unknown.

Here, we demonstrate that increased levels of a TRPC3-Nox2 complex correlate well with the severity of DOX-induced cardiac atrophy and that inhibition of TRPC3-Nox2 functional coupling suppresses DOX-induced myocardial atrophy in mice. By contrast, voluntary exercise, which induces physiological cardiac hypertrophy, reduced levels of the TRPC3-Nox2 complex. Considering that the TRPC3-Nox2 axis also mediates pressure overload-induced pathological cardiac remodeling, stable induction of the TRPC3-Nox2 complex is apparently key to the transition from adaptation to maladaptation in the heart.

\section{Results}

Deletion of TRPC3 channels attenuates DOX-induced myocardial atrophy in mice. DOX-induced congestive heart failure is characterized by a severe reduction of LV mass and systolic dysfunction, as well as bursts of ROS production in the heart (6). Treating mice with DOX caused severe reductions in heart weight and myocardial cell size as compared with vehicle-treated (sham) mice (Figure 1, A and B). DOX also significantly increased cardiac levels of TRPC3 (Figure 1C). We therefore examined the involvement of TRPC3 channels in DOX-induced heart failure using TRPC3-deficient $\left(\mathrm{TRPC}^{-/-}\right)$mice and their WT $\left(\mathrm{TRPC}^{+/+}\right)$littermates. We confirmed that the TRPC3 protein band detected in $\mathrm{TRPC}^{+/+}$hearts was not present in $\mathrm{TRPC}^{-/-}$hearts (Figure 1C). DOX elicited significant reductions in heart weight and myocardial cell size in TRPC $3^{+/+}$mice, but these effects were almost completely suppressed in $\mathrm{TRPC}^{-/-}$mice (Figure 1, D and E). Likewise, DOX-induced reductions of LV systolic and diastolic functions observed in $\mathrm{TRPC}^{+/+}$mice were canceled in $\mathrm{TRPC} 3^{-/-}$mice (Figure $1, \mathrm{~F}$ and $G$ and Tables 1 and 2). In addition, production of malondialdehyde (MDA), an index of oxidative stress, was significantly lower in DOX-treated $\mathrm{TRPC}^{-/-}$than $\mathrm{TRPC}^{+/+}$hearts (Figure $1 \mathrm{H}$ ). We confirmed that the TRPC6 mRNA expression level was never compensatively upregulated in TRPC3 ${ }^{-/-}$hearts (Supplemental Figure 1; supplemental material available online with this article; https://doi.org/10.1172/jci.insight.93358DS1). These results clearly indicate that TRPC 3 participates in DOX-induced myocardial atrophy, oxidative stress, and LV dysfunction in mice.

Deletion of TRPC3 suppresses hypoxic stress-mediated upregulation of Nox2 and myocardial atrophy in DOX-treated mice. Using hypoxyprobe, we found that DOX induced hypoxic stress in cardiac tissue (Figure $2 \mathrm{~A})$, which in turn led to increases in the abundance of hypoxia-inducible factor $1 \alpha(\mathrm{HIF} 1 \alpha)$ and heme oxygenase-1 (HO-1) in mouse hearts (Figure 2, B and C). HIF1 $\alpha$ is known to enhance production of ROS by increasing expression of Nox2 (28), and DOX also increased the abundance of Nox2 (Figure 2, B-D). The upregulation of Nox2 expression correlated well with the reduction in heart weight caused by DOX treatment (Figure 2D). This DOX-induced cardiac atrophy and Nox2 upregulation were significantly diminished in TRPC $3^{-/}$mice, and the degree to which myocardial atrophy was suppressed by TRPC 3 deletion correlated well with the Nox2 expression (Figure 2, E-G). On the other hand, TRPC3 deletion had no 
A

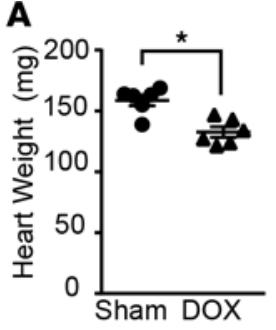

B

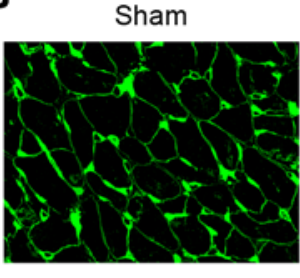

C
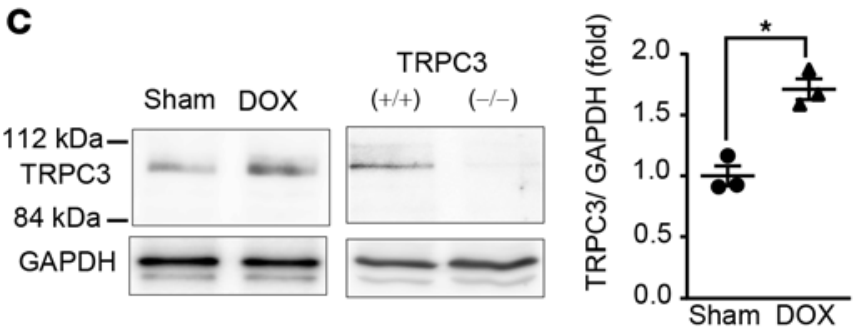

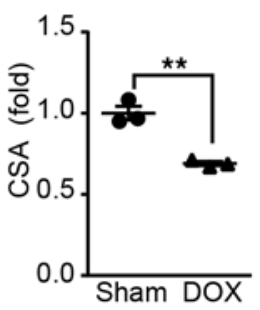

D

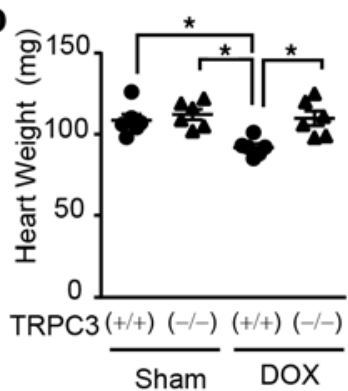

E

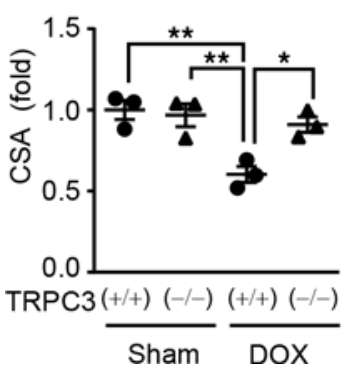

F

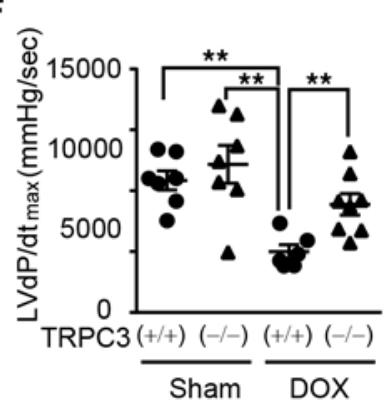

G

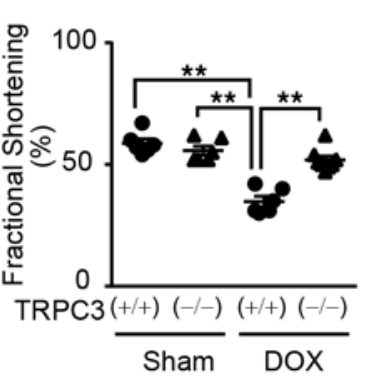

H

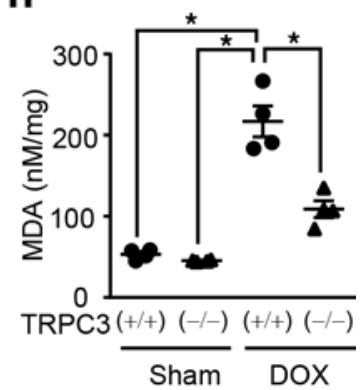

Figure 1. Prevention of DOX-induced myocardial atrophy and oxidative stress in TRPC3-deficient mouse hearts. (A and B) Decreases in heart weight (A) and cross-sectional area (CSA) of cardiomyocytes (B) in C57BL/6J mouse hearts 2 weeks after administration of vehicle (Sham) or DOX ( $n=6)$. Scale bar: $50 \mu \mathrm{m}$. (C) Abundances of TRPC3 proteins in DOX-treated C57BL/6) mouse hearts (left) and 129Sv-background TRPC $3^{+/+}$and TRPC3 ${ }^{-/-}$mouse hearts (right) $(n=3)$. (D-C) Heart weights (D), CSA (E), and LV contractility (F and $\mathbf{G})$ in 1295 v-background TRPC $3^{+/+}$and TRPC $3^{-/-}$mice treated with vehicle (Sham) or DOX $(n=6)$. (H) MDA concentrations in mouse hearts $(n=4)$. $(\mathbf{A}-\mathbf{H})$ Data are shown as the mean $\pm \mathrm{SEM}$. Significance was determined using unpaired $t$ test analysis in $\mathbf{A}-\mathbf{C}$ and one-way ANOVA followed by Tukey's comparison test in $\mathbf{D}-\mathbf{H} .{ }^{*} P<0.05,{ }^{* *} P<0.01$.

impact on the increased abundance of HIF1 $\alpha$. These data suggest that TRPC3 participates in hypoxic stress-mediated upregulation of Nox2 during DOX treatment, which leads to ROS-dependent cardiac atrophy and LV dysfunction in mice.

Formation of a TRPC3-Nox2 complex contributes to DOX-induced cardiomyocyte atrophy. TRPC 3 has two functions: activation of local $\mathrm{Ca}^{2+}$ influx-mediated $\mathrm{Ca}^{2+}$ signaling induced by physicochemical stimulation and activation of context-dependent ROS signaling through physical interaction with Nox2 (29, 30). We used neonatal rat cardiomyocytes (NRCMs) to determine whether functional coupling between TRPC3 and Nox2 is required for DOX-induced myocardial atrophy. DOX treatment decreased the size of NRCMs. TRPC3 knockdown suppressed this cardiomyocyte atrophy (Figure 3A) and increased 2',7'-dichlorofluorescein diacetate ( $\mathrm{DCFH}_{2} \mathrm{DA}$ ) fluorescence intensity (Figure 3B). Despite the high sensitivity of $\mathrm{DCFH}_{2} \mathrm{DA}$ to ROS, this probe is limited to use for measuring intracellular ROS (31). Therefore, we used a genetically encoded fluorescent sensor for hydrogen peroxide, Hyper, and confirmed that a DOX-induced increase in Hyper fluorescence intensity was suppressed by the treatment with siRNA for TRPC3 or Nox2 in H9c2 cardiac myoblasts (Supplemental Figure 2A). We previously reported that TRPC3 interacts with Nox2 via a TRPC3-specific C-terminal region - which protects Nox2 from proteasome-dependent degradation, leading to its stabilization - and that overexpression of a GFP-fused TRPC3-C terminal fragment peptide (C3-C-GFP) interfered with the interaction between Nox 2 and TRPC3 without reducing TRPC3 channel activity (20) (Figure 3C). This prompted 
Table 1. Echocardiographic parameters of $\mathrm{TRPC}^{+/+}$and $\mathrm{TRPC}^{-/-}$mice

\begin{tabular}{|c|c|c|c|c|}
\hline & $\begin{array}{c}\text { TRPC3 }^{+/+} \\
\text {Sham }(n=6)\end{array}$ & $\begin{array}{c}\text { TRPC3 }^{-/-} \\
\text {Sham }(n=6)\end{array}$ & $\begin{array}{l}\operatorname{TRPC3}^{+/+} \\
\operatorname{DOX}(n=6)\end{array}$ & $\begin{array}{l}\text { TRPC3-/- }^{-1} \\
\operatorname{DOX}(n=6)\end{array}$ \\
\hline Mortality & $0 / 6$ & $0 / 6$ & $4 / 10$ & $0 / 8$ \\
\hline BW (g) & $24.1 \pm 1.1$ & $24.3 \pm 0.8$ & $20.0 \pm 1.2^{A}$ & $22.1 \pm 1.1$ \\
\hline HR (bpm) & $523 \pm 27$ & $558 \pm 24$ & $452 \pm 30$ & $524 \pm 32$ \\
\hline IVSd (mm) & $1.0 \pm 0.1$ & $0.9 \pm 0.1$ & $0.8 \pm 0.1$ & $0.9 \pm 0.0$ \\
\hline LVPWd (mm) & $1.1 \pm 0.1$ & $1.0 \pm 0.0$ & $0.8 \pm 0.1^{\mathrm{B}}$ & $0.9 \pm 0.0$ \\
\hline EF (\%) & $92.2 \pm 0.6$ & $90.8 \pm 1.1$ & $71.0 \pm 2.4^{\mathrm{C}}$ & $88.8 \pm 1.0$ \\
\hline FS (\%) & $58.7 \pm 1.7$ & $55.7 \pm 1.7$ & $34.8 \pm 1.9^{c}$ & $51.9 \pm 1.5$ \\
\hline
\end{tabular}

BW, body weight; HR, heart rate; IVSd, interventricular septum diastolic thickness; LVPWd, left ventricular posterior wall diastolic thickness; LVIDd, left ventricular internal diameter at end-diastole; LVIDs, left ventricular internal diameter at end-systole; EF, ejection fraction; FS, fractional shortening. Data are shown as mean \pm SEM. Significance was determined by one-way ANOVA followed by Tukey's comparison test. ${ }^{A} P<0.05 \mathrm{Vs}$. TRPC ${ }^{+/+}$Sham, TRPC $3^{-/-}$ Sham. ${ }^{\mathrm{B}} P<0.05$ vs. TRPC3 ${ }^{+/+}$Sham. ${ }^{\mathrm{C}} P<0.01$ vs. TRPC3 ${ }^{+/+}$Sham, TRPC3 $^{-1-}$ Sham, TRPC3 ${ }^{-1-}$ DOX.

us to test the effect of C3-C-GFP on DOX-induced cardiomyocyte atrophy. To avoid the overlap of GFP fluorescence from DCF fluorescence, ROS production was measured using H9c2 cells expressing HA-tagged TRPC3-C terminal fragment peptide (C3-C-HA) instead of C3-C-GFP. We observed that C3-C-GFP and C3-C-HA expression significantly reduced the ROS production, cell shrinkage, Nox2 upregulation, and increase of the interaction between Nox2 and TRPC3 (Figure 3, D-F and Supplemental Figure 2B). These results suggest that functional coupling between TRPC 3 and Nox2 is essential for DOX-induced cell atrophy and ROS production in cardiac myocytes.

Cardiomyocyte-specific expression of C3-C-GFP suppresses DOX-induced cardiomyopathy. We also examined whether cardiomyocytes are cellular origins of DOX-induced cardiomyopathy in vivo. Using a cardiomyocyte-specific, cardiac troponin-T promoter-driven, C3-C-GFP-encoding adeno-associated virus, C3-C-GFP was selectively expressed in cardiomyocytes in C57BL/6J mice. Compared with control (GFP-expressing) mice, the C3-C-GFP-expressing mice showed significant suppression of the myocardial atrophy, LV dysfunction, and oxidative stress normally seen in DOX-treated mice (Figure 4, A-E, and Tables 3 and 4). We also confirmed that C3-C-GFP was widely expressed in nearly all cardiomyocytes of the LV myocardium (Figure 4F). C3-C-GFP expression significantly suppressed the increases of Nox2 and TRPC3 protein abundances in DOX-treated hearts (Figure 4G). These results suggest that cardiomyocytes are a cellular origin of the cardiac atrophy mediated via the TRPC3-Nox2 axis in DOX-treated mice.

Pharmacological inhibition of TRPC3-Nox2 interaction suppresses DOX-induced cardiomyopathy. The above results led us to consider that a compound that inhibits the TRPC3-Nox2 interaction could be an ideal

Table 2. Hemodynamic parameters of $\mathrm{TRPC}^{+/+}$and $\mathrm{TRPC}^{-/-}$mice measured by left ventricular catheter

\begin{tabular}{|c|c|c|c|c|}
\hline & $\mathrm{TRPC3}^{+/+} \operatorname{sham}(n=6)$ & TRPC3 $^{-/-} \operatorname{sham}(n=6)$ & $\mathrm{TRPC3}^{+/+} \operatorname{DOX}(n=6)$ & $\mathrm{TRPC3}^{-/-} \mathrm{DOX}(n=6)$ \\
\hline HR (bpm) & $341 \pm 18$ & $405 \pm 13$ & $361 \pm 28$ & $333 \pm 14$ \\
\hline LVESP $(\mathrm{mmHg})$ & $134 \pm 6$ & $131 \pm 5$ & $108 \pm 8$ & $122 \pm 8$ \\
\hline $\mathrm{LVdP} / \mathrm{dt}_{\text {max }}(\mathrm{mmHg} / \mathrm{s})$ & $10,835 \pm 725$ & $12,146 \pm 1,440$ & $4,991 \pm 477^{A}$ & $8,872 \pm 882$ \\
\hline $\mathrm{LVdP} / \mathrm{dt}_{\min }(\mathrm{mmHg} / \mathrm{s})$ & $7,403 \pm 551$ & $8,253 \pm 625$ & $4,657 \pm 474^{A}$ & $6,185 \pm 739$ \\
\hline$\tau(\mathrm{msec})$ & $12.6 \pm 1.2$ & $10.4 \pm 1.5$ & $18.9 \pm 1.8^{B}$ & $12.5 \pm 0.8$ \\
\hline
\end{tabular}

HR, heart rate; LVESP, left ventricular end-systolic pressure; LVEDP, left ventricular end-diastolic pressure; LVdP/dt ${ }_{\text {max }}$, left ventricular maximal rate of pressure development; $\mathrm{LVdP} / \mathrm{dt}_{\text {min }}$, left ventricular maximal rate of decay of pressure; $\tau$, monoexponential time constant of relaxation. Data are shown as mean \pm SEM. Significance was determined by one-way ANOVA followed by Tukey's comparison test. ${ }^{A} P<0.01 \mathrm{vs}$. TRPC ${ }^{+/+}$Sham, TRPC ${ }^{-/-}$Sham, TRPC ${ }^{-/-}$ DOX. ${ }^{\mathrm{B} P}<0.05$ vs. TRPC3 ${ }^{+/+}$Sham, TRPC3 $3^{-/-}$Sham, TRPC3 ${ }^{-/-}$DOX. 
A
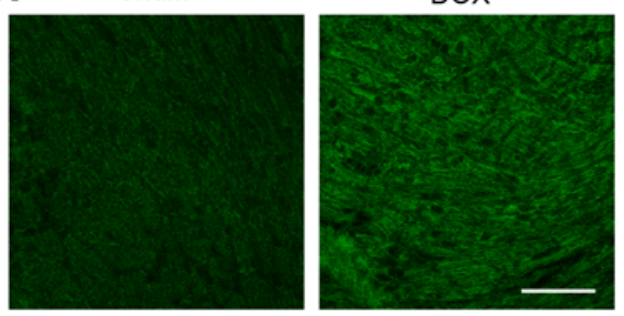

B

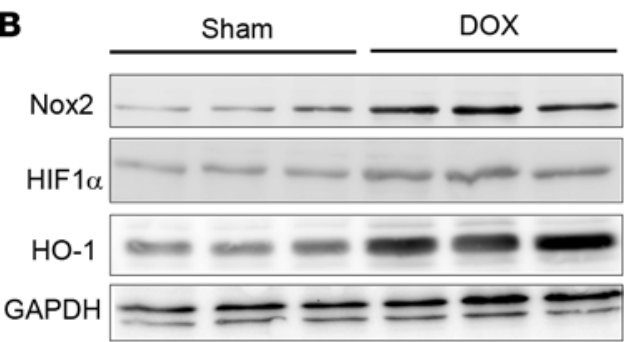

C

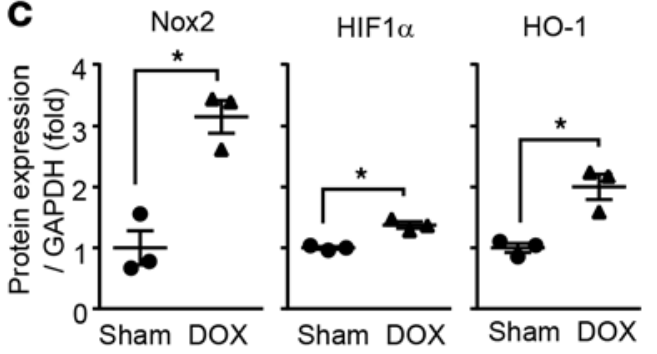

D

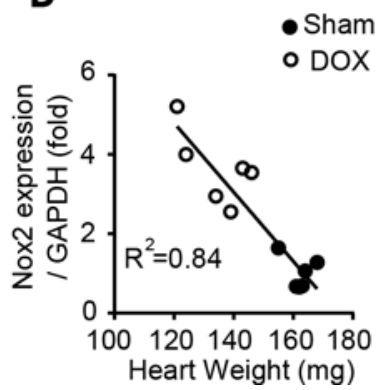

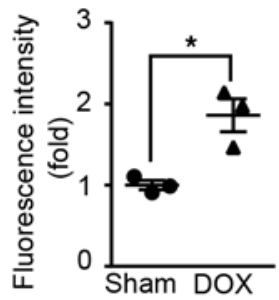

E
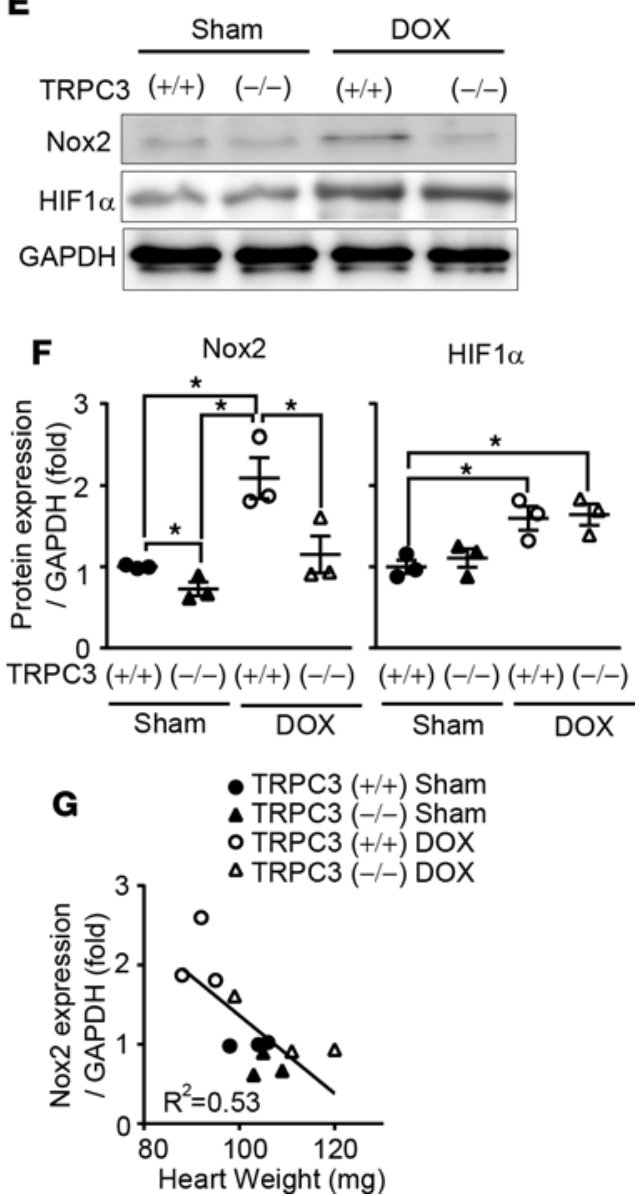

Figure 2. Requirement for TRPC3 in DOX-induced Nox2 upregulation. (A) Representative fluorescence images (left) and semiquantitative results of fluorescence intensities (right) in mouse hearts stained with hypoxyprobe $(n=3)$. Scale bar: 40 $\mu \mathrm{m}$. (B and C) Western blots (B) and quantitative results (C) for Nox2, HIF1 $\alpha$, HO-1, and CAPDH in C57BL/6] mouse hearts 2 weeks after DOX ( $n$ = 3). (D) Relationship between heart weight and Nox2 abundance. (E and F) Representative Western blots (E) and quantitative results (F) for Nox2, HIF1 $\alpha$, and GAPDH in 129Sv-background $\mathrm{TRPC}^{+/+}$and TRPC $3^{-/-}$mouse hearts $(n=3)$. (G) Relationship between heart weight and Nox2 abundance. Data are shown as the mean \pm SEM. Significance was determined using unpaired $t$ test analysis in $\mathbf{A}$ and $\mathbf{C}$, and one-way ANOVA followed by Tukey's comparison test in $\mathbf{F} .{ }^{*} P<0.05$.

drug for the treatment of heart failure. We therefore assessed the effect on the TRPC3-Nox2 interaction of six compounds previously reported to selectively inhibit TRPC 3 channel activity (Supplemental Figure 3) (32-34). Among these, pyrazole-3 (Pyr3) most effectively suppressed the DOX-induced increase in Nox2 in NRCMs (Figure 5A). Pyr3 also suppressed DOX-induced cell shrinkage, Nox2 upregulation, and ROS production in either NRCMs or H9c2 cells (Figure 5, B-D, and Supplemental Figure 4). Prolonged treatment of NRCMs with Pyr3 reduced the basal abundance of Nox2 in a concentration-dependent manner (Figure 5E) without affecting TRPC3 and Nox2 mRNA levels (Figure 5G), and Pyr3 inhibited the interaction of TRPC3 with Nox2 (Figure 5F). Pyr3 thus acted as a potent inhibitor that suppressed the TRPC3-Nox2 interaction and DOX-induced toxicity in cardiomyocytes. We further examined whether Pyr3 directly suppresses the interaction between Nox2 and TRPC 3 using cell-free system. Treatment of cell lysates with Pyr3 reduced the complex formation between Nox 2 and TRPC 3 in a concentration-dependent manner, while Pyr3 never affected the interaction between C3-C-GFP and Nox2 (Figure 5, H and I). We previously reported that Pyr3 specifically binds to extracellular N-terminal region of TRPC3 (25); these results suggest that Pyr3 inhibits the TRPC3-Nox2 complex formation by allosterically changing conformation of TRPC 3 to reduce the binding affinity to Nox2, rather than directing disturbance of the interaction between TRPC3 and Nox2. 
A

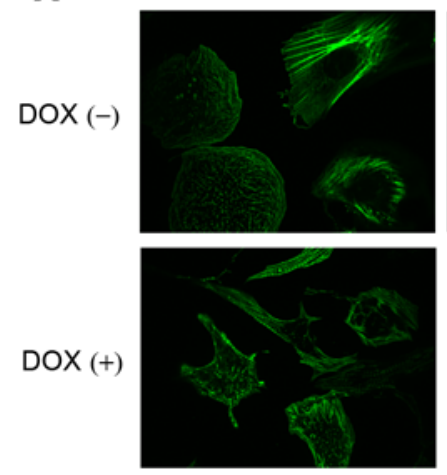

B

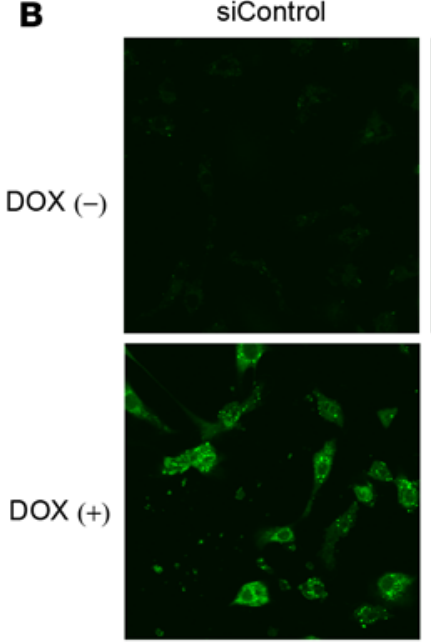

C

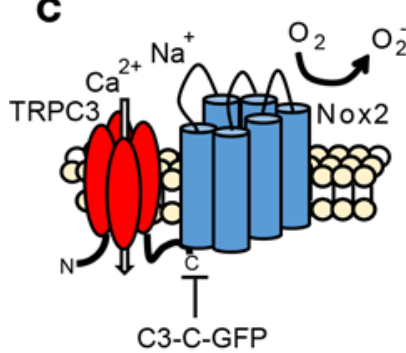

E

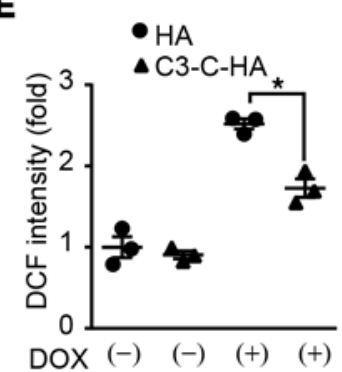

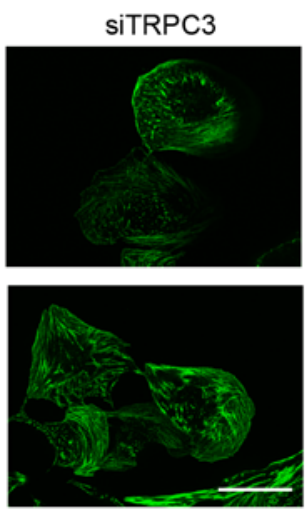

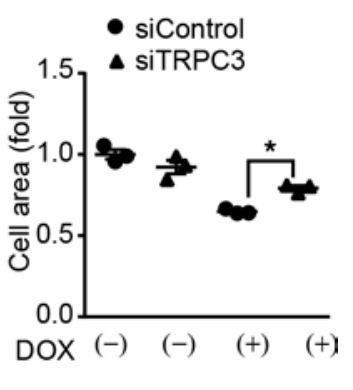

siTRPC3
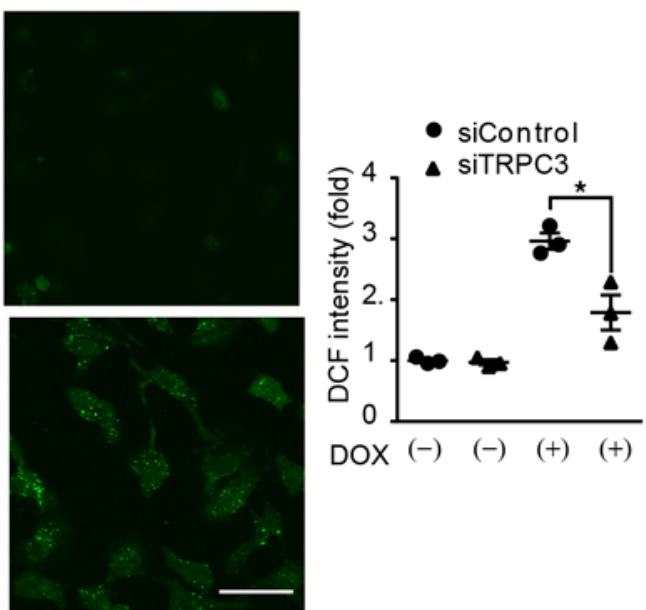

D

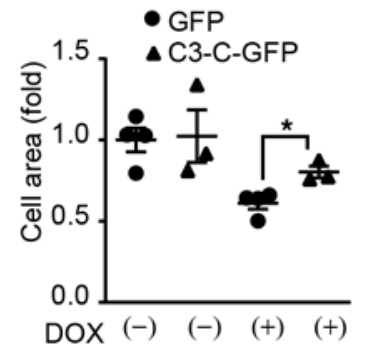

$\mathbf{F}$

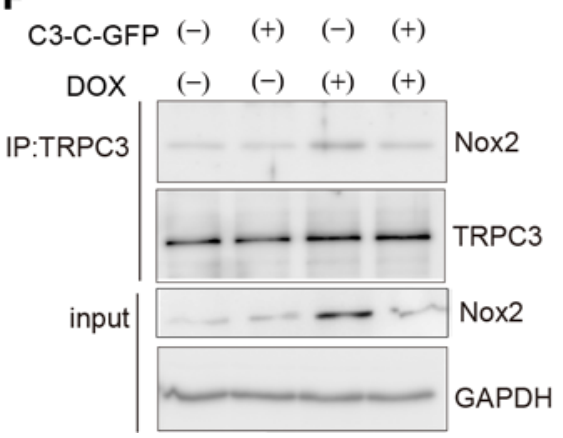

Figure 3. Inhibition of TRPC3-Nox2 coupling suppresses DOX-induced cardiomyocyte atrophy and ROS production. (A and $\mathbf{B}$ ) Effects of TRPC3-knockdown on DOX-induced cell shrinkage determined by phalloidin staining (A), and ROS production (B) in NRCMs. NRCMs were treated with DOX ( $3 \mu \mathrm{M})$ for $12 \mathrm{~h}$. Cell areas were analyzed using phalloidin staining $(n=3)$. Scale bars: $20 \mu \mathrm{m}$ (A); $40 \mu \mathrm{m}$ (B). (C) Schema for competitive disruption of the TRPC3-Nox2 protein complex by TRPC3 C-terminal minipeptide (C3-C-CFP or C3-C-HA). (D-F) Effects of C3-C-GFP on DOX-induced cardiomyocyte atrophy (D), C3-C-HA on ROS production (E), and C3-CGFP on Nox2 upregulation and interaction between TRPC3 and Nox2 (F) in H9c2 rat cardiac myoblasts ( $n=$ $3-4$ ). Data are shown as the mean \pm SEM. Significance was determined using one-way ANOVA followed by Tukey's comparison test. ${ }^{*} P<0.05$.

To test whether Pyr3 could suppress DOX-induced cardiac atrophy in vivo, osmotic pumps were used to i.p. infuse Pyr3 in C57BL/6J mice for 3 days prior to DOX treatment. The infusion of Pyr3 significantly attenuated DOX-induced decreases in LV systolic and diastolic function (Figure 5, $\mathrm{J}-\mathrm{L}$, and Tables 5 and 6), as well as the increase in MDA production and myocardial atrophy (Figure $5 \mathrm{M}$ and Supplemental Figure 5). These results suggest Pyr3 attenuates the DOX-induced myocardial atrophy in mice by destabilizing Nox 2 through disruption of the TRPC3-Nox2 interaction.

The mechanism by which DOX induces cardiotoxicity is known to differ from its antitumor actions (35). It opens the possibility of establishing a strategy for reducing or preventing DOX-induced cardiotoxicity without interfering with its antitumor effects. We therefore examined the effect of Pyr3 on DOX-induced cytotoxicity in tumor cells. A549 cells, a cell line derived from human lung adenocarcinoma, were treated with Pyr3 before DOX treatment. DOX significantly suppressed A549 cell proliferation, and Pyr3 did not inhibit the DOX-induced antitumor effect (Supplemental Figure 6). Instead, Pyr3 selectively suppressed cardiotoxicity without diminishing the antitumor effect of DOX.

Hypoxia mediates the DOX-induced upregulation of the TRPC3-Nox2 complex in cardiomyocytes. Because hypoxic stress underlies the upregulation of TRPC 3 and Nox 2 induced by DOX (36), we also investigated the contribution made by TRPC3 channels to ROS production under

hypoxia. The level of TRPC3 mRNA expression in DOX-treated hearts was 2-fold higher than that in sham hearts (Figure 6A). Similarly, levels of TRPC3 mRNA in NRCMs were increased 3-fold by hypoxic stress (Figure 6A). The level of HO-1 expression was increased under 1\% hypoxia and was not affected by Pyr3 (Figure 6B). TRPC3 inhibition appears not to diminish hypoxic stress-induced gene expression. However, Nox2 expression was actually increased by hypoxia, and TRPC 3 inhibition diminished the 

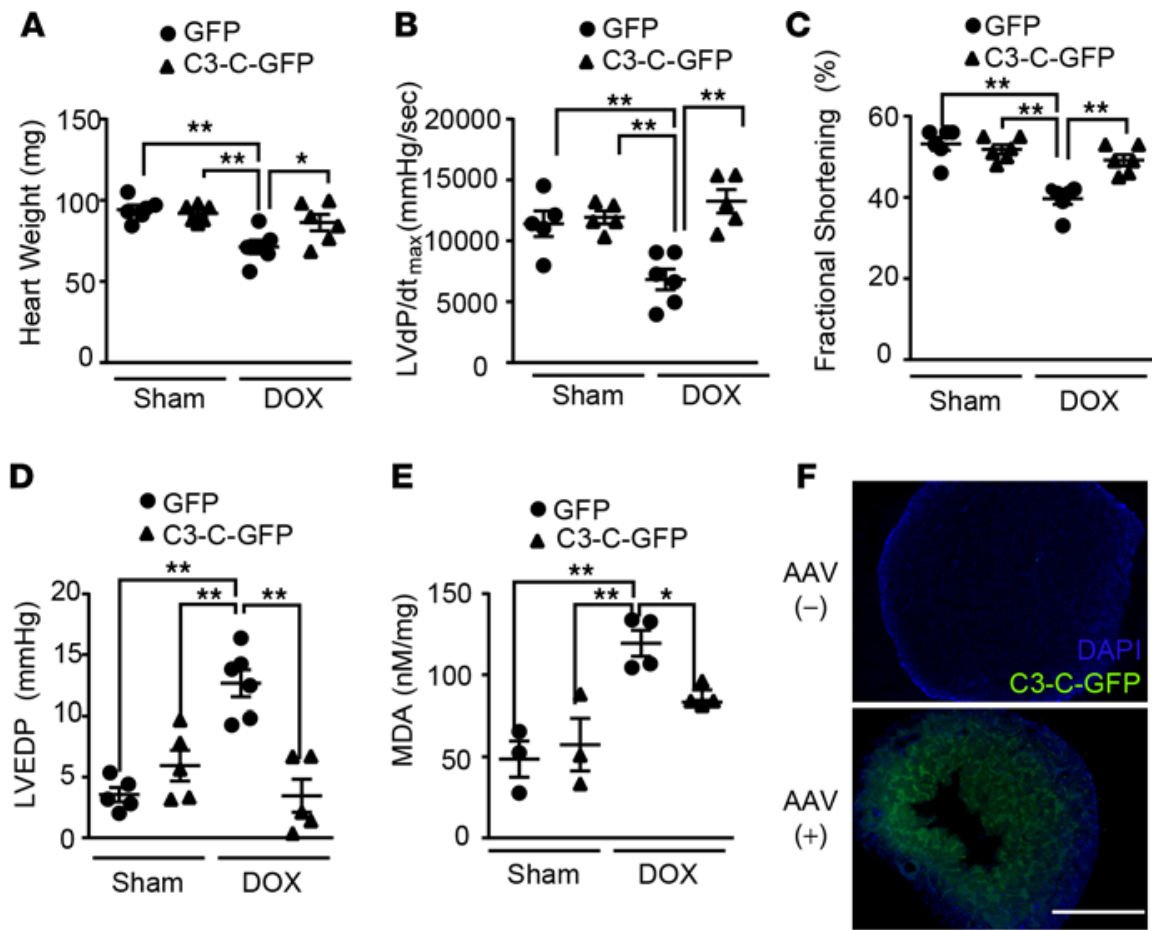

G

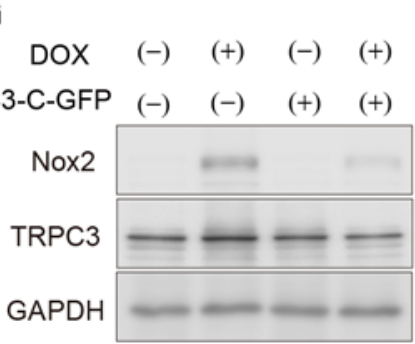

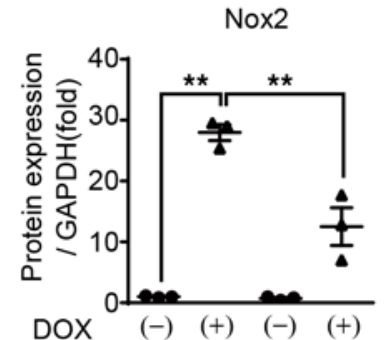

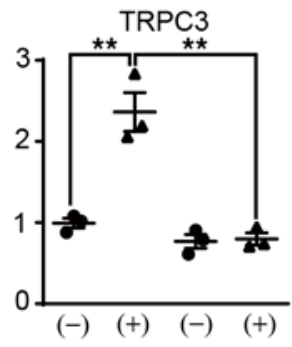

C3-C-GFP

$(-) \quad(-) \quad(+)$

(+) (+)
Figure 4. Cardiac expression of C3-C-GFP suppresses DOX-induced cardiomyopathy. (A-E) Effects of C3-C-GFP on DOX-induced reduction of heart weight (A), LV systolic (B and C) and diastolic (D) functions, and MDA production (E) in [57BL/6] mouse hearts $(n=3-6)$. (F) Representative fluorescence images of mouse hearts with or without adenoassociated virus (AAV) encoding C3-C-GFP. Scale bar: $1 \mathrm{~mm}$. (C) Effects of C3-C-GFP on protein abundances of Nox2 and TRPC3 in DOX-treated mouse hearts $(n=3)$. Data are shown as the mean \pm SEM. Significance was determined using one-way ANOVA followed by Tukey's comparison test. ${ }^{*} P<0.05,{ }^{*} P<0.01$.

hypoxia-induced Nox2 upregulation (Figure 6C). Consistent with this, siRNA-mediated silencing of TRPC 3 and Nox2 significantly suppressed hypoxia-induced ROS production determined by dihydroethidium (DHE) intensity (Figure 6D). Treating NRCMs with Pyr3 or diphenyleneiodonium (DPI), a Nox inhibitor, also reduced hypoxia-induced ROS production (Supplemental Figure 7). These results suggest that hypoxia-mediated formation of a TRPC3-Nox2 complex underlies DOX-induced ROS production in rat cardiomyocytes.

Physiological role of TRPC3 in LV diastolic function in mouse hearts. Finally, we used a pressure-volume catheter to assess whether TRPC 3 is involved in determining physiological LV contractility and flexibility. As the basal Nox2 protein abundance was slightly but significantly reduced in $\mathrm{TRPC}^{-/-}$mouse hearts (Figure 2F), the physiological role of TRPC3 was also expected to mediate mechanical stress-induced Nox2-dependent ROS signaling by stabilizing Nox2 protein in heart. Basal LV systolic function and heart rate were similar in $\mathrm{TRPC}^{+/+}$and TRPC $^{-/-}$mice (Tables 1 and 2). The slope of the end-systolic pressure volume relationship $\left(\mathrm{E}_{\text {es }}\right.$ ), an index of myocardial contractility, was similar in TRPC $3^{-/-}$and $\mathrm{TRPC}^{+/+}$hearts. By contrast, the slope of the end-diastolic pressure volume relationship $\beta$ coefficient (EDPVR $\beta$ ), an index of ventricular stiffness, was significantly lower in $\mathrm{TRPC}^{-/-}$than $\mathrm{TRPC}^{+/+}$hearts (Figure 7, A-C). Moreover, under acute volume loading, TRPC $3^{-/-}$mice exhibited significantly larger LV stroke volumes than $\mathrm{TRPC}^{+/+}$mice (Figure 7D). The ability of the heart to change its force of contraction in response to changes in venous return (i.e., the Frank-Starling mechanism) was also significantly greater in Pyr3-treated mice (Figure 7E). These results indicate that TRPC3 positively regulates LV stiffness, and inhibition or reduced expression of TRPC3 enhances myocardial flexibility in mice.

DOX-induced cardiomyopathy is reportedly attenuated by exercise (37), and exercise causes physiological hypertrophic growth of the heart (1). We therefore examined the degree to which the TRPC3-Nox2 complex mediates the three major types of cardiac plasticity: atrophy, physiological hypertrophy, and pathological hypertrophy. Voluntarily exercising mice showed a marked (10-fold) increase in daily walking distance compared with nonexercising mice (Figure 7F). Voluntary exercise for 4 weeks also caused a significant increase in heart weight with preservation of cardiac function, suggesting the development of physiological hypertrophy (Figure 7G and Table 7). Under acute volume loading, voluntarily exercising mice showed a significantly greater increase in LV stroke volume than nonexercised mice (Figure 7H). Voluntary exercise decreased the cardiac protein abundances of TRPC3 and Nox2 (Figure 7I), correlating well with the magnitude of physiological 
Table 3. Echocardiographic parameters in GFP- and C3-C-GFP-expressing mice

\begin{tabular}{|c|c|c|c|c|}
\hline & $\begin{array}{c}\text { GFP } \\
\text { Sham }(n=6)\end{array}$ & $\begin{array}{c}\text { C3-C-GFP } \\
\text { Sham }(n=6)\end{array}$ & $\begin{array}{c}\text { GFP } \\
\operatorname{DOX}(n=6)\end{array}$ & $\begin{array}{l}\text { C3-C-GFP } \\
\text { DOX }(n=6)\end{array}$ \\
\hline $\mathrm{BW}(\mathrm{g})$ & $20.5 \pm 0.4$ & $21.0 \pm 1.0$ & $17.3 \pm 1.8^{A}$ & $19.8 \pm 2.1$ \\
\hline HR (bpm) & $549 \pm 21$ & $547 \pm 25$ & $524 \pm 24$ & $528 \pm 28$ \\
\hline LVPWd (mm) & $0.8 \pm 0.0$ & $0.9 \pm 0.0$ & $0.6 \pm 0.0^{B}$ & $0.7 \pm 0.0$ \\
\hline LVIDd (mm) & $2.9 \pm 0.1$ & $2.8 \pm 0.1$ & $2.8 \pm 0.1$ & $2.9 \pm 0.1$ \\
\hline FS (\%) & $53.2 \pm 1.5$ & $51.8 \pm 2.0$ & $39.7 \pm 1.3^{\mathrm{B}}$ & $49.2 \pm 1.3$ \\
\hline
\end{tabular}

Data are shown as mean \pm SEM. Significance was determined by one-way ANOVA followed by Tukey's comparison test. ${ }^{A} P<0.05$ vs. GFP Sham, C3-C-CFP Sham. ${ }^{\mathrm{B} P}<0.05$ vs. GFP Sham, C3-C-GFP Sham, C3-C-GFP DOX.

hypertrophy in mice (Figure 7J). Voluntary exercise had no impact on the expression levels of HIF1 $\alpha$ and HO-1 proteins (Figure 7I), or on TRPC3 and Nox2 mRNAs in heart (Supplemental Figure 8), indicating that the downregulation of Nox 2 is mediated by a posttranslational mechanism. These results strongly suggest that formation of a TRPC3-Nox2 complex negatively regulates LV compliance and flexibility, which increases the risk of pathological cardiac remodeling induced by volume load.

\section{Discussion}

Although cardiac plasticity is an intrinsic compensatory mechanism to reduce hemodynamic workload, pathological atrophy or hypertrophy actually confers risk for chronic heart failure. By contrast, physiological hypertrophy, caused by hemodynamic load during postnatal growth, exercise, and pregnancy, is also a major cardiac plasticity but one in which cardiac contractility is maintained. We first demonstrated that inhibition of TRPC3 prevents DOX-induced myocardial atrophy by suppressing Nox2-mediated amplification of ROS signaling in cardiomyocytes. We also found that voluntary exercise causes hypertrophy with preserved cardiac contractility by enhancing LV compliance and flexibility through destabilization of Nox2 (Figure 7 , G-J, and Table 7). Taken together with our earlier observations made in a pressure-overloaded mouse heart model $(20,27)$, our present findings, to our knowledge, provide a new concept that a TRPC3-Nox 2 complex functions as a common major risk factor for chronic heart failure, driving pathological cardiac remodeling. Furthermore, they suggest that, by suppressing Nox2-mediated ROS production in cardiomyocytes, TRPC3 inhibition may be an effective strategy for reducing the risk of DOX-induced heart failure (Figure 7K).

Cardiac atrophy is mainly caused by sarcopenia associated with aging, cachexia, and malnutrition (2). Although it is not known whether these three major causes are involved in DOX-induced cardiac atrophy, our findings suggest the possible contribution of the TRPC3-Nox2 complex to the induction of cardiac atrophy. Future studies using animal models of sarcopenia or cancer cachexia will be required to establish the pathological role of the TRPC3-Nox2 complex formation in the cardiac atrophy seen with those ailments.

Table 4. Hemodynamic parameters in GFP- and C3-C-GFP-expressing mice measured by left ventricular catheter

\begin{tabular}{ccccc}
\hline & GFP Sham $(\boldsymbol{n}=\mathbf{5})$ & C3-C-GFP sham $(\boldsymbol{n}=\mathbf{5})$ & GFP DOX $(\boldsymbol{n}=\mathbf{6})$ & C3-C-GFP DOX $(\boldsymbol{n}=\mathbf{5})$ \\
HR $(\mathrm{bpm})$ & $458 \pm 22$ & $467 \pm 20$ & $419 \pm 33$ & $409 \pm 23$ \\
\hline LVESP $(\mathrm{mmHg})$ & $113 \pm 5$ & $126 \pm 10$ & $106 \pm 7$ & $126 \pm 7$ \\
LVEDP $(\mathrm{mmHg})$ & $3.6 \pm 0.5$ & $5.9 \pm 1.1$ & $12.7 \pm 1.0^{\mathrm{A}}$ & $3.5 \pm 1.2$ \\
\hline $\mathrm{LVdP} / \mathrm{dt}_{\max }(\mathrm{mmHg} / \mathrm{s})$ & $11,410 \pm 1,053$ & $11,930 \pm 467$ & $6,823 \pm 778^{\mathrm{A}}$ & $13,250 \pm 860$ \\
\hline $\mathrm{LVdP} / \mathrm{dt}{ }_{\min }(\mathrm{mmHg} / \mathrm{s})$ & $7,802 \pm 899$ & $8,203 \pm 1,202$ & $4,030 \pm 610^{\mathrm{B}}$ & $5,941 \pm 859$ \\
\hline$\tau$ (msec) & $9.4 \pm 0.8$ & $11.7 \pm 1.1$ & $23.5 \pm 5.1^{\mathrm{B}}$ & $11.0 \pm 0.4$
\end{tabular}

Data are shown as mean \pm SEM. Significance was determined by one-way ANOVA followed by Tukey's comparison test. ${ }^{A} P<0.01$ vs. GFP Sham, C3-C-GFP Sham, C3-C-GFP DOX. ${ }^{B} P<0.05$ vs. GFP Sham, C3-C-GFP Sham, C3-C-GFP DOX. 
A

$\operatorname{DOX} \quad(-) \quad(+) \quad(+) \quad(+) \quad(+) \quad(+) \quad(+) \quad(+)$ DMSO DMSO Pyr3 Pyr2 2910 SAR 8009 GSK Nox2 GAPDH

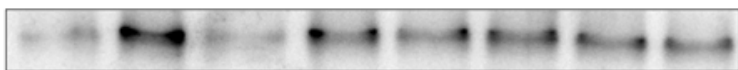

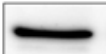

$\mathbf{B}$

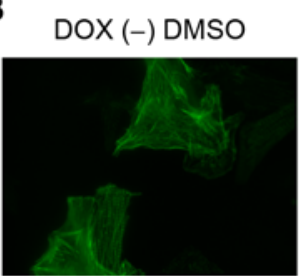

C $\operatorname{DOX}(-)$ DMSO

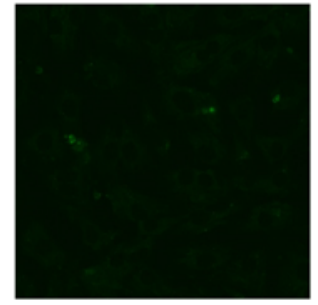

D
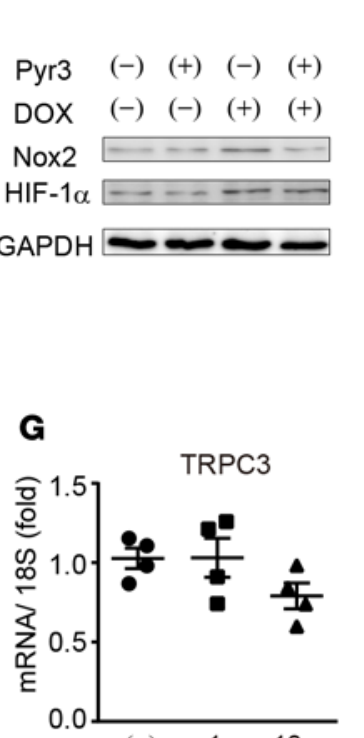

Pyr3 ( $\mu$ M) $(-) \quad 1 \quad 10$

J
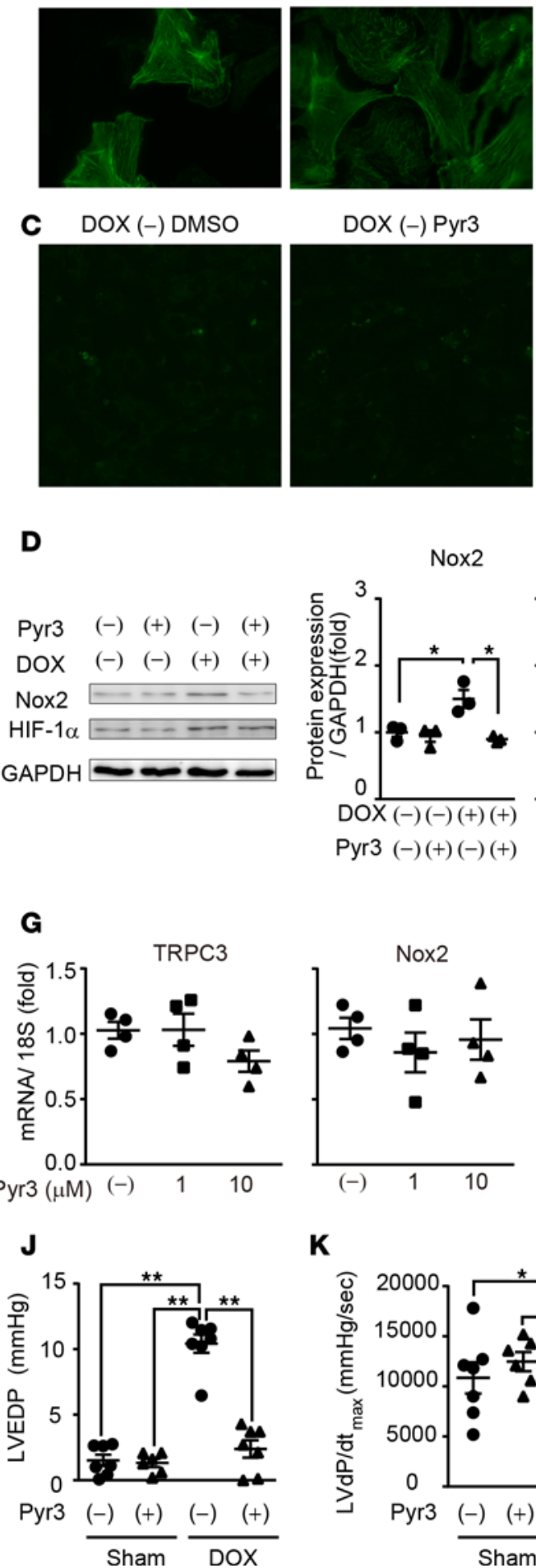

DOX (-) Pyr3

DOX (-) Pyr3

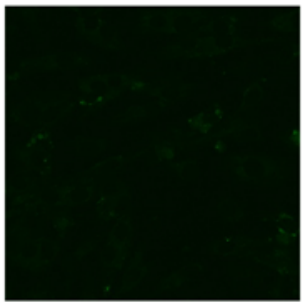

Nox2
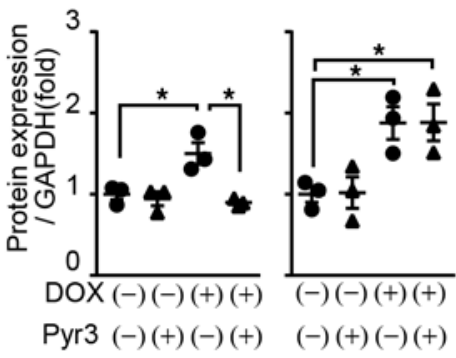

$(-)(+)(-)(+)$ Nox2

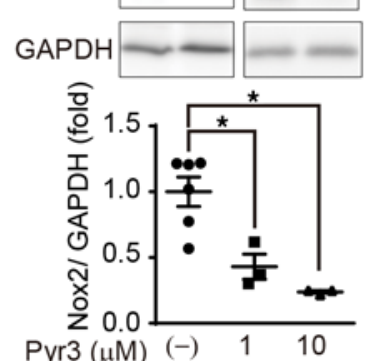

H Pyr3
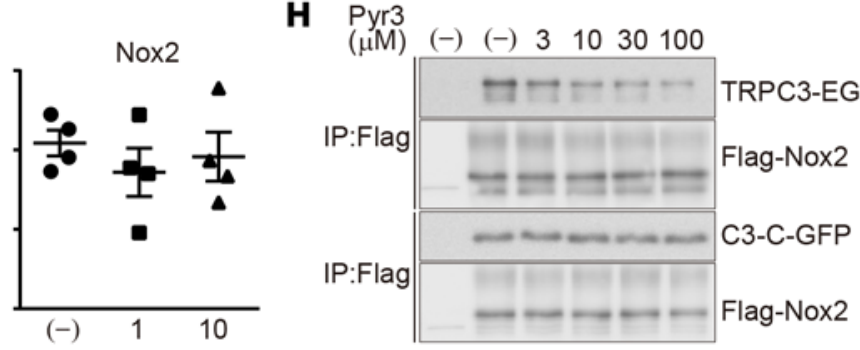

K

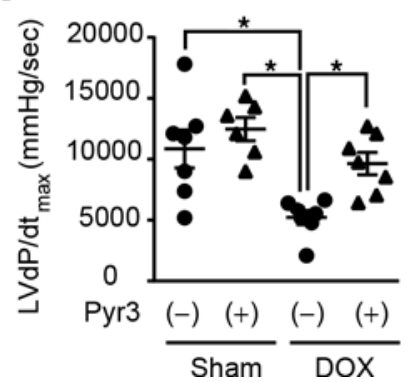

L

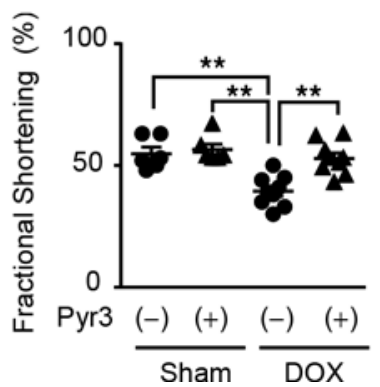

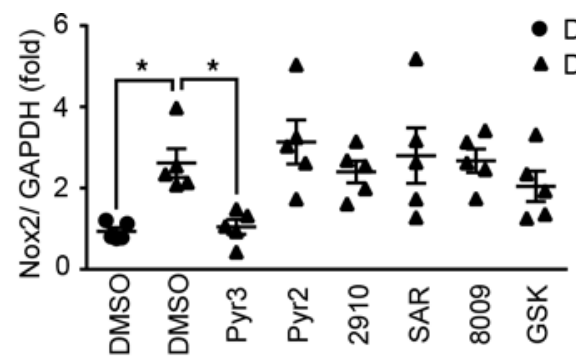

- DOX (-)

DOX (+)
E $\begin{array}{r}\text { Pyr3 } \\ (\mu \mathrm{M})\end{array}$

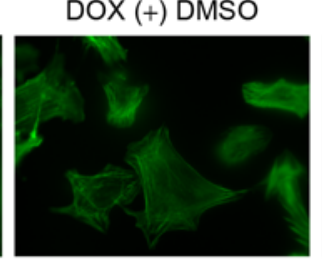

\section{DOX (+) DMSO}

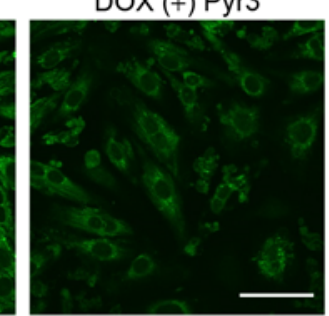

(-) $1 \quad(-) 10$
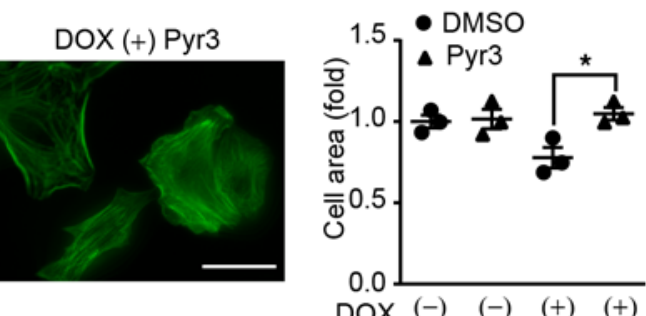

$\operatorname{Dox}(-) \quad(-) \quad(+) \quad(+)$

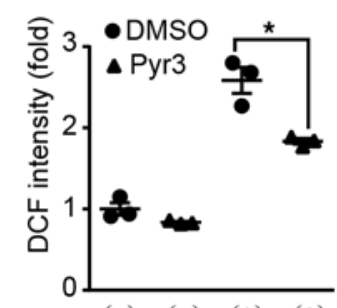

DOX (-) (-) (+) (+)

F
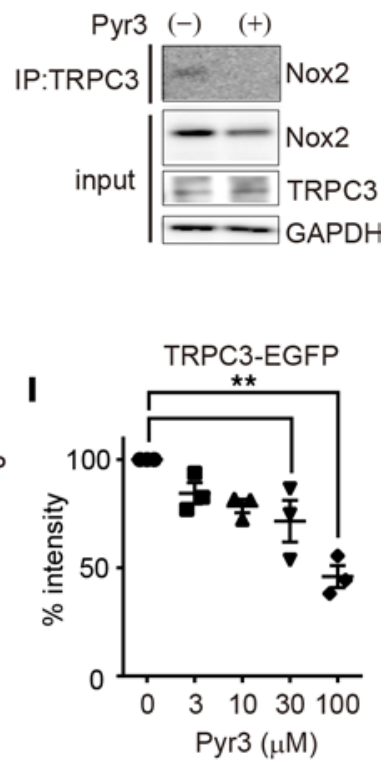

M

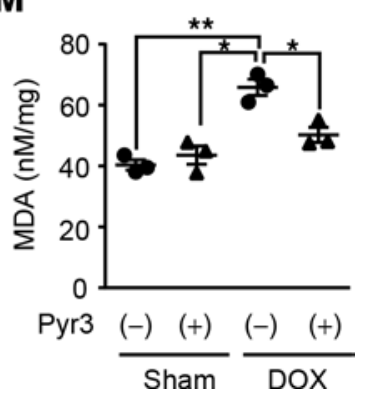


Figure 5. Pharmacological inhibition of TRPC3-Nox2 interaction suppresses DOX-induced cardiomyopathy. (A) Effects of TRPC3 inhibitors on DOX-induced Nox2 upregulation in NRCMs. NRCMs were treated with the indicated TRPC3 inhibitor $(1 \mu \mathrm{M}) 30$ min prior to DOX treatment $(3 \mu \mathrm{M}$ for $12 \mathrm{~h}, n=5)$. (B-D) Effects of pyrazole-3 (Pyr3) on DOX-induced atrophy (B) and ROS production (C), and upregulation of Nox2 and HIF1 $\alpha$ proteins (D) in NRCMs. NRCMs were treated with Pyr3 $(1 \mu \mathrm{M}) 30$ min prior to DOX (3 $\mu \mathrm{M}$ for $12 \mathrm{~h}, n=3$ ). Scale bar: $20 \mu \mathrm{m}$ (B); $40 \mu \mathrm{m}$ (C). (E) Abundances of Nox2 proteins in NRCMs with or without Pyr3 treatment for $36 \mathrm{~h}(n=3)$. (F) Effect of Pyr3 $(10 \mu \mathrm{M})$ on endogenous interaction between TRPC3 and Nox2 in NRCMs ( $n=3)$. (G) Expression levels of TRPC3 and Nox2 mRNAs in NRCMs with or without Pyr3 treatment for $36 \mathrm{~h}(n=4)$. (H and I) Effect of Pyr3 on the interaction between Nox2 and either TRPC3-EGFP or C3-C-GFP in cell-free system. Representative Western blot (H) and quantification of TRPC3-EGFP interacting with Nox2 (I) $(n=3)$. (J-L) Effect of Pyr3 on DOX-induced LV dysfunction in C57BL/6J mice $(n=6-7)$. (M) Effect of Pyr3 on DOX-induced MDA production ( $n=3)$. Data are shown as the mean \pm SEM. Significance was determined using one-way ANOVA followed by Tukey's comparison test. ${ }^{*} P<0.05,{ }^{* *} P<0.01$.

Anthracyclines, including DOX, are inexpensive, highly effective antitumor drugs used to treat breast cancer, lung cancer, lymphoma, and leukemia. However, the cardiotoxicity caused by anthracyclines limits their utility. In the present study, we revealed the molecular mechanism underlying the initiation of DOX-induced cardiomyopathy and suggest that inhibiting the TRPC3-Nox2 complex could be an effective strategy to prevent/reverse DOX-induced chronic heart failure. In this study, we demonstrated that Pyr3, a compound that can inhibit TRPC3 channel activity (25), potently prevented DOX-induced myocardial atrophy and LV dysfunction in mice (Figure 5, Tables 5 and 6, Supplemental Figure 5). Pyr3 was also found to suppress DOX-induced ROS production by destabilizing Nox 2 protein through disrupting the TRPC3-Nox 2 complex formation in NRCMs (Figure 5). As Pyr3 reportedly inhibits several other channels, such as CRAC channels and TREK and TASK2 potassium channels $(24,38)$, it cannot be simply assumed that the effects of Pyr3 are mediated by disrupting the TRPC3-Nox 2 interaction. However, our results clearly show that pharmacological effects by Pyr3 are quite similar to those by knockdown or genetic deletion of TRPC3 in cardiomyocytes. Pyr3 had no impact on upstream factors regulating the TRPC3-Nox2 complex formation, such as upregulation of HIF1 $\alpha$ protein and basal mRNA levels of TRPC 3 and Nox2. We also revealed that Pyr3 directly acted on the complex formation between Nox2 and full-length TRPC3, but not the C-terminal fragment of TRPC3, in a cell-free system (Figure 5, H and I), suggesting the involvement of allosteric conformational changes in TRPC 3 for disruption of the TRPC3-Nox2 complex. These findings strongly support the idea that Pyr3 prevents DOX-induced Nox2 upregulation by disrupting the TRPC3-Nox2 complex formation. Other approaches that reportedly prevent or mitigate DOX-induced dilated cardiomyopathy include exercise (37); treatment with cilostazol (39), an inhibitor of phosphodiesterase type 3 (PDE3), antioxidants (10), or iron chelators (40); and expression of $\mathrm{G} \alpha_{\mathrm{q}}$-inhibitory peptide in cardiomyocytes (41). We previously observed that inhibition OF PDE3 (42) or $\mathrm{G}_{q}$-mediated phosphatidylinositol turnover (22) attenuates TRPC3/6 channel activity, and we observed here that exercise reduces TRPC 3 expression in mouse hearts (Figure 7I). These findings are consistent with our new finding that TRPC 3 in cardiomyocytes is a critical mediator of DOX-induced dilated cardiomyopathy.

Although the pathological significance of the TRPC3-Nox2 complex in DOX-induced myocardial atrophy in mice is evident, the physiological role of TRPC 3 remains elusive. Redox/cysteine modification of proteins that regulate $\mathrm{Ca}^{2+}$ handling has been reported to affect cardiac contractility (43), including Nox2-depending ROS signaling (X-ROS signaling) in the diastolic filling heart (19). Actually, TRPC3-deficient mice had less protein abundances of TRPC 3 and Nox2, as well as less basal MDA

Table 5. Echocardiographic parameters of mice with Pyr3 administration.

\begin{tabular}{|c|c|c|c|c|}
\hline & Veh sham $(n=6)$ & Pyr3 sham $(n=6)$ & Veh DOX $(n=9)$ & Pyr3 DOX $(n=9)$ \\
\hline BW (g) & $23.9 \pm 0.5$ & $23.5 \pm 0.5$ & $20.1 \pm 0.5^{A}$ & $21.2 \pm 0.4$ \\
\hline HR (bpm) & $471 \pm 10$ & $429 \pm 14$ & $425 \pm 8$ & $435 \pm 22$ \\
\hline LVPWd (mm) & $1.0 \pm 0.0$ & $1.0 \pm 0.1$ & $0.7 \pm 0.0^{\mathrm{B}}$ & $0.8 \pm 0.0$ \\
\hline LVIDd (mm) & $2.6 \pm 0.1$ & $2.6 \pm 0.0$ & $2.6 \pm 0.1$ & $2.5 \pm 0.1$ \\
\hline LVIDs (mm) & $1.1 \pm 0.1$ & $1.1 \pm 0.1$ & $1.6 \pm 0.1^{\complement}$ & $1.2 \pm 0.1$ \\
\hline
\end{tabular}

Data are shown as mean \pm SEM. Significance was determined by one-way ANOVA followed by Tukey's comparison test. ${ }^{A} P<0.05$ vs. Veh Sham, Pyr3 Sham. ${ }^{B} P<0.05$ vs. Veh Sham, Pyr3 Sham, Pyr3 DOX. ${ }^{C} P<0.01$ vs. Veh Sham, Pyrz Sham, Pyr3 DOX. 

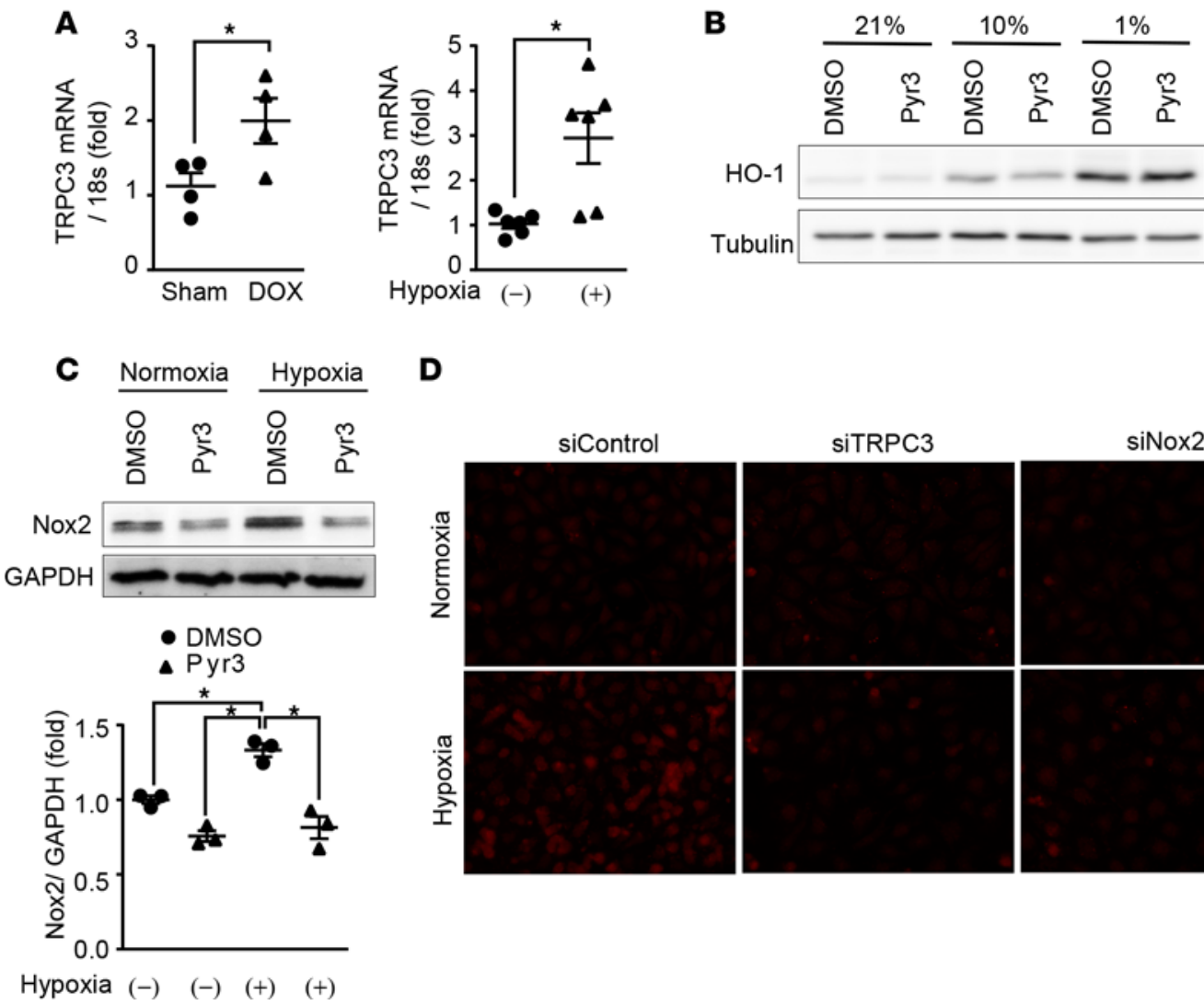
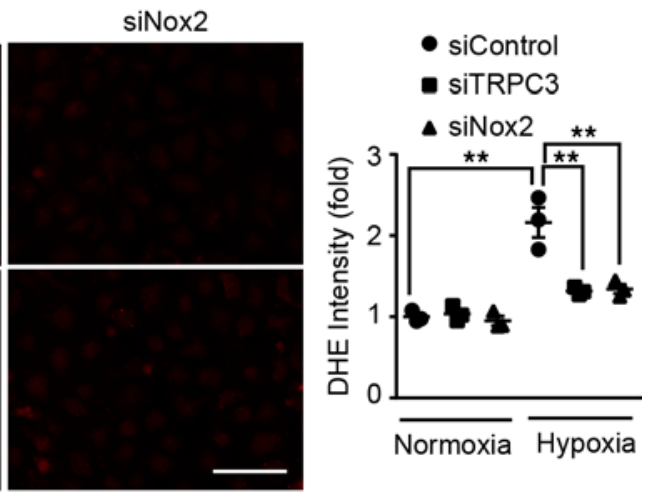

Figure 6. Hypoxic stress mediates DOX-induced upregulation of TRPC3-Nox2 complex in cardiomyocytes. (A) Levels of TRPC3 mRNA expression in DOX-treated C57BL/6) mouse hearts $(n=4)$ and hypoxia-exposed NRCMs $(n=6)$. (B) Abundances of H0-1 and tubulin in NRCMs exposed to $10 \%$ or $1 \%$ hypoxia with or without Pyr3 (300 nM, $n=3$ ). (C) Effects of Pyr3 on hypoxia-induced Nox2 upregulation $(n=3)$. (D) Effects of siRNAs for TRPC3 or Nox2 on hypoxia-induced ROS production. NRCMs were exposed to $1 \%$ hypoxia for $12 \mathrm{~h}$ and then treated with dihydroethidium (DHE, $2 \mu \mathrm{M}$ ) for $1 \mathrm{~h}$. NRCMs were transfected with siRNAs $72 \mathrm{~h}$ before hypoxia $(n=3)$. Scale bar: $50 \mu \mathrm{m}$. Data are shown as the mean \pm SEM. Significance was determined using one-way ANOVA followed by Tukey's comparison test. ${ }^{*} P<0.05,{ }^{* *} P<0.01$.

content in the heart (Figure 1, C and H, and Figure 2F). This implies that the TRPC3-Nox2 complex formation underlies TRPC3-mediated negative regulation of LV compliance in both physiological and pathological conditions. This idea is supported by the fact that voluntary exercise, an effective treatment to prevent the heart from DOX-induced cardiotoxicity, decreased protein abundances of Nox2 and TRPC3 (Figure 7I). As the phenotypic change of mouse heart induced by voluntary exercise seems consistent with those in TRPC3-deficient mice, we suggest that TRPC3 is a prerequisite for the basal expression of Nox 2 because it prevents constitutive degradation in cardiac cells. Although Nox2-mediated ROS production induced by physiological stretching of cardiomyocytes (i.e., X-ROS signaling) is reported to participate in enhancement of cardiac contractility, Nox2 deletion has no impact on cardiac compliance compared with the impact by TRPC3 inhibition (13). This indicates that Nox2-derived ROS signaling has two roles: one is to promote cardiac contractility through oxidative modification of $\mathrm{Ca}^{2+}$-handling proteins, and the other is to suppress cardiac compliance through unknown mechanism(s). Our results suggest that the context-dependent ROS signaling mediated by the TRPC3-Nox2 complex plays a key role in cardiac stiffness. Future identification of a new TRPC3-mediated ROS target protein will elucidate its underlying mechanism.

The mechanism by which exercise downregulates TRPC 3 and Nox2 is also obscure. As a DOX-induced increase in TRPC3 mRNA level was associated with upregulation of HIF1 $\alpha$, we first thought that exercise treatment downregulates TRPC 3 and Nox 2 mRNAs by suppressing hypoxic stress and/or HIF1 $\alpha$ upregulation. However, exercise had no impact on basal HIF1 $\alpha$ abundance, which was comparable with that in sedentary mice (Figure 7I). Exercise treatment never decreased the expression levels of TRPC 3 and Nox 2 mRNAs (Supplemental Figure 8), but it significantly reduced TRPC3 and Nox2 protein abundances (Figure 7I). This 
Table 6. Hemodynamic parameters of mice with Pyr3 administration measured by left ventricular catheter

\begin{tabular}{|c|c|c|c|c|}
\hline & Veh Sham $(n=6)$ & Pyr3 Sham $(n=6)$ & Veh DOX $(n=7)$ & Pyr3 DoX $(n=7)$ \\
\hline HR (bpm) & $392 \pm 30$ & $415 \pm 16$ & $352 \pm 21$ & $342 \pm 8$ \\
\hline LVESP $(\mathrm{mmHg})$ & $100 \pm 6$ & $116 \pm 6$ & $100 \pm 8$ & $115 \pm 5$ \\
\hline $\mathrm{LVdP} / \mathrm{dt}_{\text {max }}(\mathrm{mmHg} / \mathrm{s})$ & $10,866 \pm 1,441$ & $12,467 \pm 811$ & $5,221 \pm 535^{\mathrm{B}}$ & $9,643 \pm 849$ \\
\hline $\mathrm{LVdP} / \mathrm{dt}_{\min }(\mathrm{mmHg} / \mathrm{s})$ & $5,606 \pm 599$ & $5,823 \pm 263$ & $3,810 \pm 449^{B}$ & $5,510 \pm 343$ \\
\hline$\tau(\mathrm{msec})$ & $10.9 \pm 1.7$ & $11.1 \pm 1.0$ & $23.4 \pm 3.4^{A}$ & $13.9 \pm 1.4$ \\
\hline
\end{tabular}

Data are shown as mean \pm SEM. Significance was determined by one-way ANOVA followed by Tukey's comparison test. ${ }^{A} P<0.01$ vs. Veh Sham, Pyr3 Sham, Pyr3 DOX. ${ }^{\mathrm{B}} P<0.05$ vs. Veh Sham, Pyr3 Sham, Pyr3 DOX.

result strongly suggests the involvement of posttranslational modulation or modification of TRPC3 or Nox2 proteins in the TRPC3-Nox2 stable complex formation. Further studies for identification of some intrinsic molecule(s) that can disrupt the interaction between TRPC3 and Nox2 from exercised heart will be required.

All $\mathrm{Ca}^{2+}$ transporters present on the myocardial plasma membrane are generally thought to participate in positive inotropic and/or chronotropic responses to physiochemical stimuli. Although several reports, including one from our group, have demonstrated the pathological effects of TRPC channels in vivo in rodent hearts (44), TRPC channels are believed to be hardly expressed in normal hearts and to be uninvolved in normal cardiac physiology, as TRPC KO had no impact on cardiac contractility or heart rate in mice. In the context of our in vitro finding that TRPC3 mediates mechanical stressinduced background $\mathrm{Ca}^{2+}$ entry in cardiomyocytes (20), our present observation using TRPC3-deficient mice suggests TRPC 3 contributes to the negative regulation of myocardial compliance and flexibility in response to volume load. We anticipate that these findings will provide the basis for a new strategy to maintain the cardiac performance while driving cardiac plasticity.

\section{Methods}

Mice, chemicals, and reagents. The 129Sv mice with homozygous deletion of the gene-encoding TRPC3 were provided by the National Institute of Environmental Health Sciences. Genotyping was performed using PCR the primers TRPC3-A (5'-GAATCCACCTGCTTACAACCATGTG-3') and TRPC3-B (5'-GGTGGAGGTAACACACAGCTAAGCC-3'). The PCR was performed using Phusion High-Fidelity DNA polymerase (Thermo Scientific). C57BL/6J mice were purchased from Japan SLC Inc. Mice were maintained in a specific-pathogen-free area under a 12-hour/12-hour light/dark cycle. Male 7-week-old mice were administered DOX (15 mg/kg, Wako) i.p. and sacrificed 2 weeks later. Osmotic pumps (ALZET) for sustained administration of Pyr3 (30 $\mu \mathrm{g} / \mathrm{kg} /$ day) were implanted i.p. 3 days before DOX administration. The TRPC3/6 blocker, GSK-417651A, was purchased from Focus Biomolecules. Other TRPC3/6 inhibitors were synthesized or made to order as described previously (25, 32-34). Cell lines (H9c2 and A549) were purchased from American Type Culture Collection (ATCC).

Voluntary exercise experiment in mice. Male 8-week-old mice (C57BL/6J)

Table 7. Echocardiographic parameters of mice with voluntary exercise were exposed to voluntary exercise in a cage with a running wheel. The mice were allowed to use the voluntary running wheel for 4 weeks. Nonexercised (sedentary) mice were kept in identical cages with an immovable wheel. Spontaneous activity was recorded every minute and walking distances were summed up for 24 hours using Actimo-100 (Shinfactory).

Transthoracic echocardiography and cardiac catheterization. Echocardiography and cardiac catheterization were performed as described previously $(15,20)$. Echocardiography was performed using a Nemio-XG echocardiograph (Toshiba) with a 14-MHz transducer after anesthetizing the mice using a mixture of domitor (Zenoaq), midazolam (Sando), and butorphanol (Meiji Seika Pharma). LV hemodynamic parameters were assessed after DOX treatment in the anesthetized mice using a micronanometer catheter (Millar 1.4F, SPR 671, Millar Instruments). unpaired $t$ test. ${ }^{A} P<0.01$ vs. Sedentary. 
A

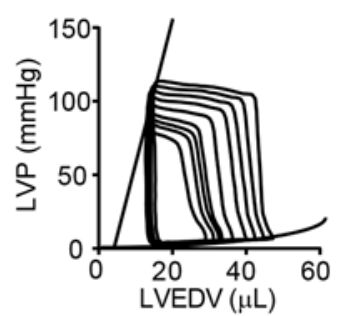

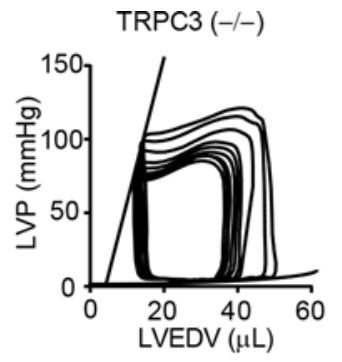

B

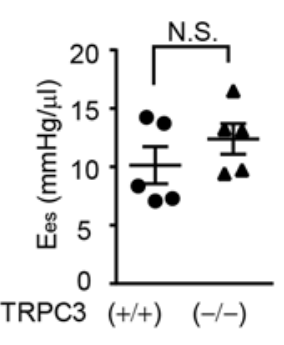

C

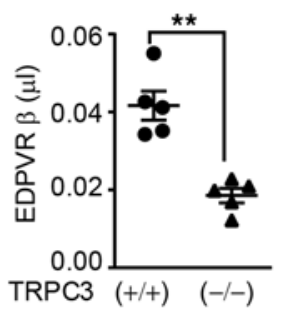

D

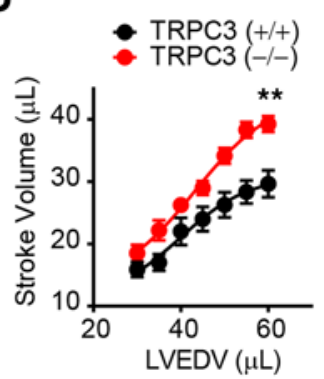

E

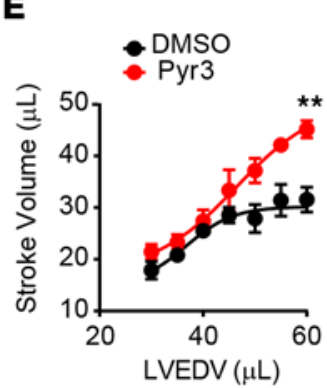

$\mathbf{F}$

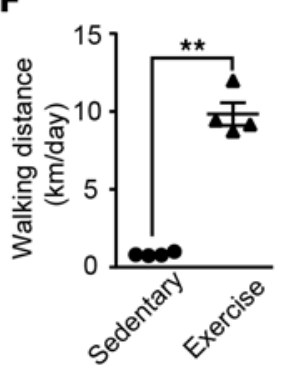

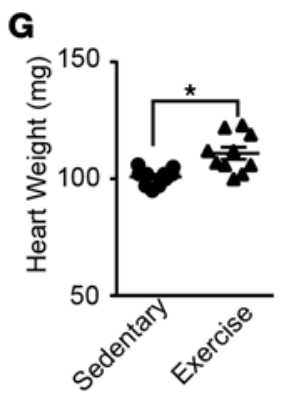

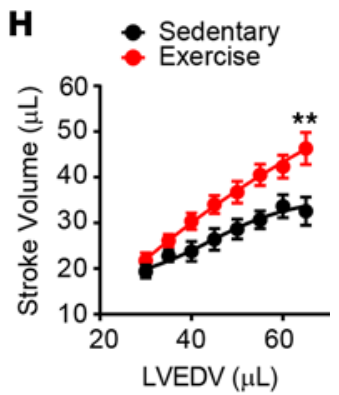

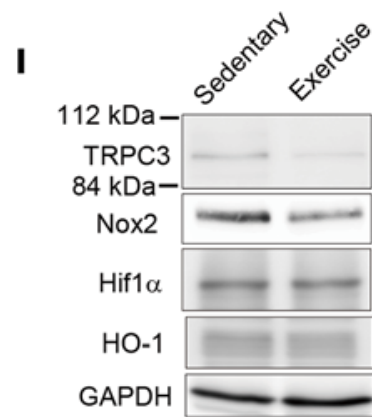
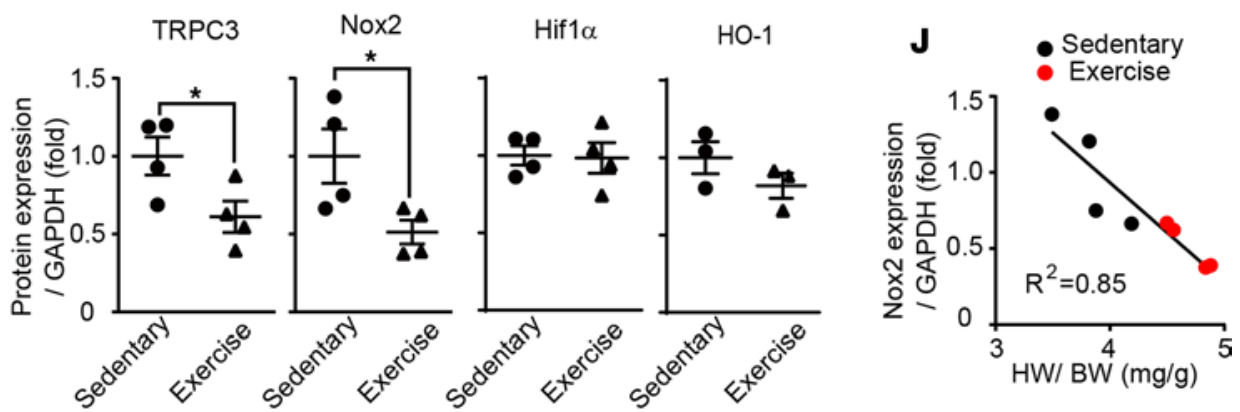

K

$$
\text { LV compliance, flexibility }
$$

\section{Pathological hypertrophy}

TRPC3-Nox2 expression
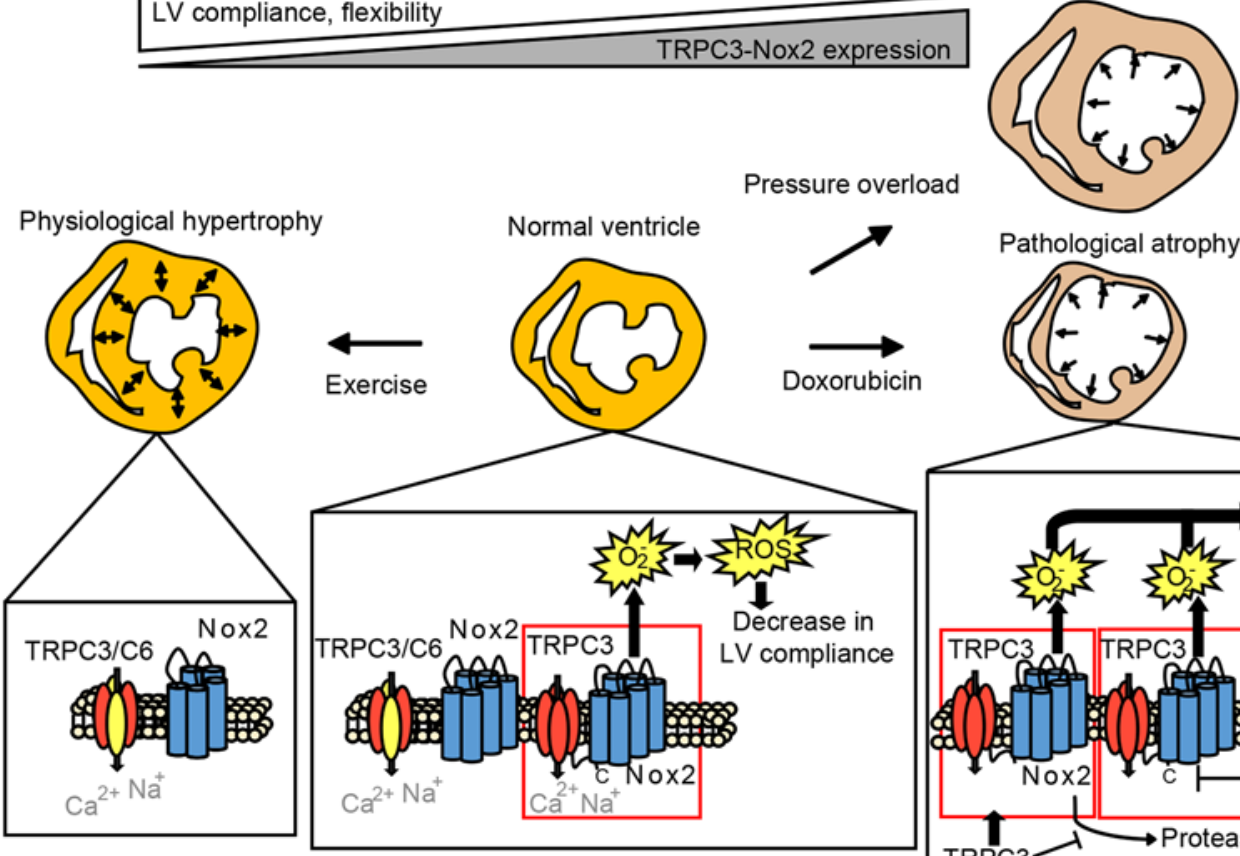

Pressure overload

Pathological atrophy

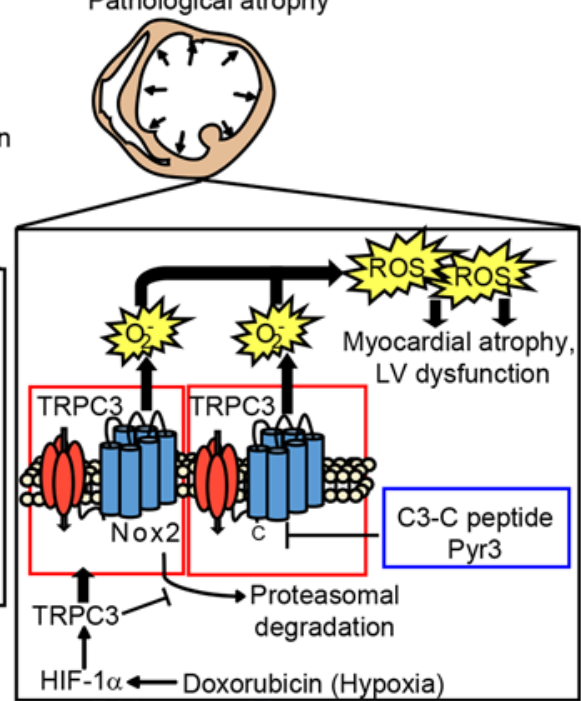

Figure 7. Roles of TRPC3-Nox2 complex in myocardial flexibility and plasticity. (A) Representative LV pressure-volume loops after inferior vena cava occlusion in TRPC $3^{+/+}$and TRPC3 ${ }^{-/-}$mice. (B and C) Results of LV systolic indexes (end-systolic elastance; $\mathrm{E}_{\mathrm{es}}$ ) (B) and diastolic indexes (end-diastolic pressure-volume relationship $\beta$; EDPVR $\beta)(C)(n=5)$. (D) Cardiac performance under volume overload reflecting the Frank-Starling law in TRPC $3^{+/+}$and TRPC $3^{-/-}$ 
mice $(n=6)$. (E) Cardiac performance under volume overload in [57BL/6) mice treated with Pyr3 $(n=4)$. (F) Spontaneous walking activity in C57BL/6) mice with and without a running wheel $(n=4)$. (G-J) Effects of voluntary exercise on heart weight $(\mathbf{G})(n=10)$, LV performance under volume load $(\mathbf{H})(n=7)$, and protein abundances of TRPC3, Nox2, HIF1 $\alpha$, and HO-1 (I) ( $n=3-4)$, and relationship between heart weight and Nox2 expression (J). Data are shown as the mean \pm SEM. Significance was determined using unpaired $t$ tests in $\mathbf{B}, \mathbf{C}, \mathbf{F}$, and $\mathbf{G}$ and two-way ANOVA followed by Bonferroni's comparison test in $\mathbf{D}$, $\mathbf{E}$, and $\mathbf{H} .{ }^{*} P<0.05,{ }^{* *} P<0.01$. (K) Schema for the role of TRPC3 channels in cardiac plasticity. The TRPC3-Nox2 axis restricts volume-loaded LV diastolic filling through ROS production in normal hearts. Increases in the abundance of the TRPC3-Nox2 complex underlie pathological hypertrophy and atrophy. Voluntary exercise can induce physiological hypertrophy under hemodynamic load by reducing the TRPC3-Nox2 complex.

In vivo pressure-volume analysis. Pressure-volume analysis was performed according to established protocols $(45,46)$. Pyr3 (100 $\mu \mathrm{g} / \mathrm{kg} /$ day) was administered using an osmotic minipump for 7 days before the analysis. Each mouse was anesthetized (induction: 3\%-4\% isoflurane, maintenance: $1 \%-2 \%$ isoflurane), intubated, and mechanically ventilated (MiniVent, model 845, Harvard Apparatus) with $100 \% \mathrm{O}_{2}$. The animal was then placed on a temperature-controlled heating platform to maintain body temperature. After accessing the internal jugular vein using a 30-gauge needle connected to polyethylene tubing, $12.5 \%$ albumin in normal saline was infused at $300 \mu \mathrm{l} /$ hour after an initial $50-\mu 1$ bolus to counteract the peripheral vasodilation and hypotension caused by the anesthesia (46). The diaphragm and ribs were then carefully dissected using an electrocautery device (Bovie Medical), the LV apex was punctured with a 27-gauge needle, and a catheter (Millar 1.4F, SPR 839, Millar Instruments) was introduced through the puncture. Mice were allowed to stabilize for 5 minutes, after which transient occlusion of inferior vena cava was performed to calculate $\mathrm{E}_{\mathrm{es}}$ for evaluation of loadindependent systolic function, and EDPVR $\beta$ was determined to evaluate end-diastolic stiffness $(45,46)$. $\mathrm{E}_{\mathrm{es}}$ was calculated as ESP $=\mathrm{E}_{\mathrm{es}} \times \mathrm{ESV}+\mathrm{V}_{0}$, and EDPVR $\beta$ was calculated as EDP $=\alpha \times \exp ^{\beta \times \mathrm{EDV}}$, where ESP represents end-systolic pressure, ESV represents end-systolic volume, $\mathrm{V}_{0}$ represents calculated pressure when LV volume equals $0, \alpha$ represents the stiffness and scaling coefficient, and EDV represents end-diastolic volume. To investigate the cardiac responsiveness to volume overload, $12.5 \%$ albumin was transfused at $2 \mathrm{ml} /$ hour. Hypertonic saline $(10 \% \mathrm{NaCl}, 10 \mu \mathrm{l})$ was injected to calculate parallel conductance.

$A A V$-mediated expression of TRPC3 C-terminal fragment in mouse hearts. The Nox2-interacting fragment from the TRPC3 C-terminus (C3-C fragment) was PCR amplified and cloned into pEGFP-N1 vector. The EGFP-fused C3-C fragment (C3-C-GFP) cassette under the control of cardiac troponin T promoter was subcloned into pZac2.1 (Penn Vector). Viral vectors encoding EGFP or C3-C-GFP were generated as described previously (47). The AAV vectors $\left(1 \times 10^{10}\right.$ genomic copies $)$ were i.p. injected into male 6-day-old C57BL/6J mice. Expression of C3-C-GFP in the LV myocardium was verified using a fluorescence microscope (BZ-X710, Keyence).

Morphological analysis. Hearts were removed from mice, washed in PBS to remove the blood, and fixed by $10 \%$ neutral buffered formalin. To assess (cross-sectional area) CSA in the cardiomyocytes, sections were stained with wheat germ agglutinin (WGA) conjugated with Alexa Fluor 488 (Invitrogen) and observed using a fluorescence microscope. Five LV regions were selected at random for each specimen, and the average values were calculated using ImageJ software (NIH).

Assessment of oxidative stress in vivo. Total cardiac MDA was assessed in mice using a Bioxytech MDA-586 kit (OxisResearch) according to the manufacturer's instructions, as described previously (15, 20). Briefly, frozen mouse heart samples were homogenized in potassium phosphate extraction (KPE) buffer ( $\mathrm{pH} 7.5$ ) containing $100 \mathrm{mM}$ potassium phosphate, $5 \mathrm{mM}$ EDTA, 0.1\% (v/v) Triton X-100, 0.6\% sulfosalicylic acid, and $5 \mathrm{mM}$ buthylated hydroxytoluene (OxisResearch). The resultant lysates were clarified by centrifugation at $20,400 \mathrm{~g}$ for 10 minutes at $4^{\circ} \mathrm{C}$. Samples of the supernatant were then allowed to react with $\mathrm{N}$-methyl-2-phenylindole for 1 hour at an acidic $\mathrm{pH}$ at $45^{\circ} \mathrm{C}$, after which the samples were clarified by centrifugation at 20,400 $\mathrm{g}$ for 10 minutes at $4^{\circ} \mathrm{C}$ and read at $586 \mathrm{~nm}$ using a Spectra Max i3 (Molecular Devices).

Real-time PCR. Total RNA was isolated from frozen mouse heart samples using an RNeasy Fibrous Tissue Mini Kit (Qiagen) or from cardiomyocytes using an RNeasy Mini Kit (Qiagen) according to the manufacturer's instructions. Quantitative real-time PCR was performed using an ABI PRISM 7500 Real-Time PCR System (Applied Biosystems) with OneStep RT-PCR Kit (Qiagen) or Power SYBR green PCR master mix (Applied Biosystems) according to the manufacturer's instructions. The information of Taqman probes and primer sets were as previously described (20).

Western blotting and IP. Mouse hearts were homogenized in RIPA buffer containing $0.1 \%$ SDS, $0.5 \%$ sodium deoxycholate, $1 \% \mathrm{NP}-40,150 \mathrm{mM} \mathrm{NaCl}, 50 \mathrm{mM}$ Tris- $\mathrm{HCl}$ (pH 7.4), and protease inhibitor cocktail (Nacalai). Samples $(10 \mu \mathrm{g})$ were then fractionated by SDS-PAGE and transferred onto PVDF membranes (Millipore). The objective proteins were detected using the indicated antibodies. After incubation 
with the secondary antibody, the bands were visualized using Western Lightning Plus ECL (PerkinElmer). Images were captured with an ImageQuant LAS 4000 (GE healthcare Life Science) and quantified using ImageQuant TCL software (GE Healthcare Life Sciences). Primary antibodies against Nox2 (1:1,000, sc-130543, Santa Cruz Biotechnology Inc.), $\alpha \beta$-tubulin (1:2,000, 2143, Cell Signaling Technology), GAPDH (1:2,000, sc-25778, Santa Cruz Biotechnology Inc.), HIF1 $\alpha$ (1:1,000, NB100-105, Novus Biologicals), and HO-1 (1:1,000, ADI-SPA-895, Enzo) were used.

To analyze expression of endogenous Nox2 in vitro and in vivo, the total membrane fraction was isolated as described previously (30). To investigate the abundance of TRPC3, endogenous TRPC3 was concentrated by IP. Briefly, TRPC3 proteins were immunoprecipitated from mouse heart lysates (500 $\mu \mathrm{g}$ total protein), cell lysates from Pyr3-treated NRCM, or cell lysates from DOX-treated H9c2 cells using antiTRPC3 antiserum $(1.5 \mu \mathrm{g})$ obtained from guinea pig (20) and protein G sepharose beads (GE Healthcare). After incubation for 24 hours, the immune complexes were washed 3 times with lysis buffer, suspended in SDS sample buffer containing 0.1 M DTT, and incubated for 1 hour at room temperature. TRPC3 was detected with rabbit anti-TRPC3 antibody (1:1,000, ACC-016, Alomone). For the IP with Pyr3 in a cell-free system, HEK293 cells expressing Flag-tagged Nox2 and either TRPC3-EGFP or C3-C-GFP were lysed in 1\% Triton X-100, $140 \mathrm{mM} \mathrm{NaCl}, 1 \mathrm{mM}$ EDTA, $20 \mathrm{mM}$ Tris- $\mathrm{HCl}$ (pH 7.4), and protease inhibitor cocktail (Nacalai). Equal amounts of cell lysates were aliquoted to 5 tubes and incubated with Flag M2 agarose for 12 hours to immunoprecipitate Flag-Nox2 in the presence of Pyr3 at the indicated concentration. Immune complexes were washed 3 times with the lysis buffer and eluted with $0.1 \mathrm{mg} / \mathrm{ml}$ Flag peptide (Sigma-Aldrich) in lysis buffer.

Detection of hypoxia using a hypoxyprobe kit. Hypoxia in mouse hearts was detected using a hypoxyprobe kit (Hypoxyprobe HPI Inc.) according to the manufacturer's instructions. Hypoxyprobe solution $(60 \mathrm{mg} / \mathrm{kg})$ was i.p. injected 60 minutes prior to sacrifice. Frozen tissue was cut into $4-\mu \mathrm{m}-$ thick sections and incubated first in $1 \%$ BSA in PBS for 1 hour at $4^{\circ} \mathrm{C}$ and then with anti-hypoxyprobe antibody (1:50 dilution) in $1 \% \mathrm{BSA}$ in PBS overnight at $4^{\circ} \mathrm{C}$. After washing, the sections were incubated with secondary antibody for 1 hour at room temperature. Sections were then observed using a confocal laser microscopy, and fluorescence intensity was analyzed using ImageJ (NIH) software.

Isolation of cardiomyocytes from neonatal rat. Cardiomyocytes were isolated from neonatal rats as described previously (48). For TRPC3 and Nox2 knockdown, the cells were transfected with siRNAs (20 nM; ref. 20) for 72 hours using Lipofectamine RNAiMAX (Thermo Fisher scientific).

Measurements of cell size and ROS production in rat cardiac cells. NRCMs and H9c2 cardiac myoblasts were visualized by Alexa Fluor 488 Phalloidin (Thermo Fisher Scientific), and cell surface area was observed using a fluorescence microscope and analyzed in at least 50 cells in each experiment using ImageJ software. Production of ROS in NRCMs and H9c2 cardiac myoblasts was measured using DCF$\mathrm{H}_{2} \mathrm{DA}$ as described previously (15). Cells were loaded with $\mathrm{DCFH}_{2} \mathrm{DA}(10 \mu \mathrm{M})$ for 10 minutes at $37^{\circ} \mathrm{C}$. The DCF fluorescence excited by illumination at $488 \mathrm{~nm}$ was observed at room temperature using a confocal laser microscopy. For the detection of hydrogen peroxide, H9c2 cells were transfected with cDNA encoding Hyper (Evrogen) by electroporation (2 pulses of 1,200 mV for 10 milliseconds; Neon Transfection System, Invitrogen). After 24-hour recovery, cells were plated on glass-base dishes, cultured for another 24 hours, and treated with DOX for 12 hours. Hyper was excited at $488 \mathrm{~nm}$, and emissions were collected at $536 \mathrm{~nm}$. Images of single cell fluorescence were collected (40 cells per experiment) and analyzed by Metafluor (Molecular Device). DHE staining of siRNA-transfected NRCMs was performed by the incubation with DHE $(2 \mu \mathrm{M})$ for 1 hour. Cells $\left(2 \times 10^{5}\right.$ cells $/ 35 \mathrm{~mm}$ dish $)$ were fixed in $2 \%$ paraformaldehyde for 10 minutes to stop the reaction. To avoid the possibility of cell population dependency, the averaged DHE fluorescence intensity was introduced by averaging the DHE florescence intensity of 40-50 cells in each experiment.

Tumor cell viability. Tumor cell viability was analyzed using an MTT assay kit (Sigma Aldrich). A549 cells were treated with DOX $(10 \mu \mathrm{M})$ with or without Pyr3 $(3 \mu \mathrm{M})$ for 24 hours. Cells were further incubated in MTT-containing $(50 \mu \mathrm{g} / \mathrm{ml})$ medium for 6 hours and then lysed with DMSO. The absorbance at 570 nm was measured using Spectra Max i3.

Statistics. All results are presented as the mean \pm SEM from at least 3 independent experiments. Statistical comparisons were made using unpaired $t$ tests for two-group comparisons or using one-way ANOVA with Tukey's post hoc test or two-way ANOVA with Bonferroni's post hoc test for comparisons among 3 or more groups. 
Study approval. All protocols using animals were approved by the ethics committees at the National Institutes of Natural Sciences or the Animal Care and Use Committee and were performed according to the institutional guidelines concerning the care and handling of experimental animals.

\section{Author contributions}

$\mathrm{MN}$ supervised and conceived the project; TS designed experiments, interpreted data, and prepared the manuscript; TS, TNT, T. Ide, AN, and RM performed experiments; SO, SH, T. Ito, NK, KU, HS, and YM contributed reagents/analytic tools; and $\mathrm{MN}$ edited the manuscript.

\section{Acknowledgments}

$\mathrm{TRPC}^{-1-}$ mice were provided from Lutz Birnbaumer of the NIEHS, Research Triangle Park, North Carolina, USA. This research was funded by grants from the Japan Science and Technology Agency; Precursory Research for Embryonic Science and Technology Program (JPMJPR1336 to MN); Grants-in-Aid for Scientific Research (25293018 and 16776376 to MN); Scientific Research on Innovative Areas (Research in a Proposed Research Area 'Oxygen Biology' [14430291 to MN] and 'Living in Space' [16H01656 to TNT]); and Platform for Drug Discovery, Informatics, and Structural Life Science (to MN) from the Ministry of Education, Culture, Sports, Science, and Technology of Japan.

Address correspondence to: Motohiro Nishida, Division of Cardiocirculatory Signaling, Okazaki Institute for Integrative Bioscience (National Institute for Physiological Sciences), National Institutes of Natural Sciences, Higashiyama 5-1, Myodaiji-cho, Okazaki, Aichi 444-8787, Japan. Phone: 81.564.59.5560; Email: nishida@nips.ac.jp.

1. Frey N, Katus HA, Olson EN, Hill JA. Hypertrophy of the heart: a new therapeutic target? Circulation. 2004;109(13):1580-1589.

2. Hill JA, Olson EN. Cardiac plasticity. N Engl J Med. 2008;358(13):1370-1380.

3. Yeh ET, et al. Cardiovascular complications of cancer therapy: diagnosis, pathogenesis, and management. Circulation 2004;109(25):3122-3131.

4. Witteles RM, Bosch X. Myocardial Protection During Cardiotoxic Chemotherapy. Circulation. 2015;132(19):1835-1845.

5. Zhang S, et al. Identification of the molecular basis of doxorubicin-induced cardiotoxicity. Nat Med. 2012;18(11):1639-1642.

6. Burridge PW, et al. Human induced pluripotent stem cell-derived cardiomyocytes recapitulate the predilection of breast cancer patients to doxorubicin-induced cardiotoxicity. Nat Med. 2016;22(5):547-556.

7. Felker GM, et al. Underlying causes and long-term survival in patients with initially unexplained cardiomyopathy. $N$ Engl $\mathrm{J}$ Med. 2000;342(15):1077-1084.

8. Zhu W, et al. Acute doxorubicin cardiotoxicity is associated with p53-induced inhibition of the mammalian target of rapamycin pathway. Circulation. 2009;119(1):99-106.

9. Cove-Smith L, et al. An integrated characterization of serological, pathological, and functional events in doxorubicin-induced cardiotoxicity. Toxicol Sci. 2014;140(1):3-15.

10. Ghigo A, Li M, Hirsch E. New signal transduction paradigms in anthracycline-induced cardiotoxicity. Biochim Biophys Acta. 2016;1863(7 Pt B):1916-1925.

11. Singal PK, Iliskovic N. Doxorubicin-induced cardiomyopathy. N Engl J Med. 1998;339(13):900-905.

12. Singal PK, Iliskovic N, Li T, Kumar D. Adriamycin cardiomyopathy: pathophysiology and prevention. FASEB J. 1997;11(12):931-936

13. Zhao Y, et al. Nox2 NADPH oxidase promotes pathologic cardiac remodeling associated with Doxorubicin chemotherapy. Cancer Res. 2010;70(22):9287-9297.

14. Zorov DB, Filburn CR, Klotz LO, Zweier JL, Sollott SJ. Reactive oxygen species (ROS)-induced ROS release: a new phenomenon accompanying induction of the mitochondrial permeability transition in cardiac myocytes. J Exp Med. 2000;192(7):1001-1014.

15. Kitajima N, et al. TRPC3-mediated Ca2+ influx contributes to Rac1-mediated production of reactive oxygen species in MLP-deficient mouse hearts. Biochem Biophys Res Commun. 2011;409(1):108-113.

16. Ago T, Kuroda J, Pain J, Fu C, Li H, Sadoshima J. Upregulation of Nox4 by hypertrophic stimuli promotes apoptosis and mitochondrial dysfunction in cardiac myocytes. Circ Res. 2010;106(7):1253-1264.

17. Li JM, Gall NP, Grieve DJ, Chen M, Shah AM. Activation of NADPH oxidase during progression of cardiac hypertrophy to failure. Hypertension. 2002;40(4):477-484.

18. Lassègue B, San Martín A, Griendling KK. Biochemistry, physiology, and pathophysiology of NADPH oxidases in the cardiovascular system. Circ Res. 2012;110(10):1364-1390.

19. Prosser BL, Ward CW, Lederer WJ. X-ROS signaling: rapid mechano-chemo transduction in heart. Science. 2011;333(6048):1440-1445.

20. Kitajima N, et al. TRPC3 positively regulates reactive oxygen species driving maladaptive cardiac remodeling. Sci Rep. 2016;6:37001.

21. Montell C, Rubin GM. Molecular characterization of the Drosophila trp locus: a putative integral membrane protein required for phototransduction. Neuron. 1989;2(4):1313-1323.

22. Onohara N, et al. TRPC3 and TRPC6 are essential for angiotensin II-induced cardiac hypertrophy. EMBO J. 
2006;25(22):5305-5316.

23. Dietrich A, Kalwa H, Fuchs B, Grimminger F, Weissmann N, Gudermann T. In vivo TRPC functions in the cardiopulmonary vasculature. Cell Calcium. 2007;42(2):233-244.

24. Seo K, et al. Combined TRPC3 and TRPC6 blockade by selective small-molecule or genetic deletion inhibits pathological cardiac hypertrophy. Proc Natl Acad Sci USA. 2014;111(4):1551-1556.

25. Kiyonaka S, et al. Selective and direct inhibition of TRPC3 channels underlies biological activities of a pyrazole compound Proc Natl Acad Sci USA. 2009;106(13):5400-5405.

26. Wu X, Eder P, Chang B, Molkentin JD. TRPC channels are necessary mediators of pathologic cardiac hypertrophy. Proc Natl Acad Sci USA. 2010;107(15):7000-7005.

27. Numaga-Tomita T, et al. TRPC3-GEF-H1 axis mediates pressure overload-induced cardiac fibrosis. Sci Rep. 2016;6:39383.

28. Diebold I, Petry A, Sabrane K, Djordjevic T, Hess J, Görlach A. The HIF1 target gene NOX2 promotes angiogenesis through urotensin-II. J Cell Sci. 2012;125(Pt 4):956-964.

29. Nishida $\mathrm{M}$, et al. Amplification of receptor signalling by $\mathrm{Ca} 2+$ entry-mediated translocation and activation of PLCgamma2 in B lymphocytes. EMBO J. 2003;22(18):4677-4688

30. Numaga T, et al. Ca2+ influx and protein scaffolding via TRPC3 sustain PKCbeta and ERK activation in B cells. JCell Sci. 2010;123(Pt 6):927-938.

31. Griendling KK, et al. Measurement of Reactive Oxygen Species, Reactive Nitrogen Species, and Redox-Dependent Signaling in the Cardiovascular System: A Scientific Statement From the American Heart Association. Circ Res. 2016;119(5):e39-e75.

32. Urban N, Hill K, Wang L, Kuebler WM, Schaefer M. Novel pharmacological TRPC inhibitors block hypoxia-induced vasoconstriction. Cell Calcium. 2012;51(2):194-206.

33. Schleifer $\mathrm{H}$, et al. Novel pyrazole compounds for pharmacological discrimination between receptor-operated and store-operated $\mathrm{Ca}(2+)$ entry pathways. Br J Pharmacol. 2012;167(8):1712-1722

34. Maier T, et al. Discovery and pharmacological characterization of a novel potent inhibitor of diacylglycerol-sensitive TRPC cation channels. Br J Pharmacol. 2015;172(14):3650-3660.

35. Minotti G, Menna P, Salvatorelli E, Cairo G, Gianni L. Anthracyclines: molecular advances and pharmacologic developments in antitumor activity and cardiotoxicity. Pharmacol Rev. 2004;56(2):185-229.

36. Chu W, et al. Mild hypoxia-induced cardiomyocyte hypertrophy via up-regulation of HIF-1 $\alpha$-mediated TRPC signalling. JCell Mol Med. 2012;16(9):2022-2034.

37. Scott JM, Khakoo A, Mackey JR, Haykowsky MJ, Douglas PS, Jones LW. Modulation of anthracycline-induced cardiotoxicity by aerobic exercise in breast cancer: current evidence and underlying mechanisms. Circulation. 2011;124(5):642-650.

38. Kim HJ, Woo J, Nam Y, Nam JH, Kim WK. Differential modulation of TWIK-related K(+) channel (TREK) and TWIK-related acid-sensitive $\mathrm{K}(+)$ channel 2 (TASK2) activity by pyrazole compounds. Eur J Pharmacol. 2016;791:686-695.

39. Koh JS, et al. Protective effect of cilostazol against doxorubicin-induced cardiomyopathy in mice. Free Radic Biol Med. 2015;89:54-61.

40. Spagnuolo RD, Recalcati S, Tacchini L, Cairo G. Role of hypoxia-inducible factors in the dexrazoxane-mediated protection of cardiomyocytes from doxorubicin-induced toxicity. Br J Pharmacol. 2011;163(2):299-312.

41. Lu XL, et al. Gaq protein carboxyl terminus imitation polypeptide GCIP-27 improves cardiac function in chronic heart failure rats. PLoS One. 2015;10(3): $\mathrm{e} 0121007$.

42. Nishioka K, et al. Cilostazol suppresses angiotensin II-induced vasoconstriction via protein kinase A-mediated phosphorylation of the transient receptor potential canonical 6 channel. Arterioscler Thromb Vasc Biol. 2011;31(10):2278-2286.

43. Breitkreuz M, Hamdani N. A change of heart: oxidative stress in governing muscle function? Biophys Rev. 2015;7(3):321-341.

44. Nishida M, Kurose H. Roles of TRP channels in the development of cardiac hypertrophy. Naunyn Schmiedebergs Arch Pharmacol. 2008;378(4):395-406.

45. Pacher P, Nagayama T, Mukhopadhyay P, Bátkai S, Kass DA. Measurement of cardiac function using pressure-volume conductance catheter technique in mice and rats. Nat Protoc. 2008;3(9):1422-1434.

46. Cingolani OH, Kass DA. Pressure-volume relation analysis of mouse ventricular function. Am J Physiol Heart Circ Physiol. 2011;301(6):H2198-H2206.

47. Masuda T, et al. Dorsal horn neurons release extracellular ATP in a VNUT-dependent manner that underlies neuropathic pain. Nat Commun. 2016;7:12529.

48. Nishida M, et al. Galpha12/13-mediated up-regulation of TRPC6 negatively regulates endothelin-1-induced cardiac myofibroblast formation and collagen synthesis through nuclear factor of activated T cells activation. J Biol Chem. 2007;282(32):23117-23128 